\title{
Decomposed Sliding Mode Control of the Drive with Interior Permanent Magnet Synchronous Motor and Flexible Coupling
}

\author{
Jan Vittek ${ }^{1}$ and Sergey Ryvkin ${ }^{2}$ \\ ${ }^{1}$ University of Zilina, Univerzitna 1, 01026 Zilina, Slovakia \\ ${ }^{2}$ Trapeznikov Institute of Control Sciences, Profsojuznaja 65, 117997 Moscow, Russia \\ Correspondence should be addressed to Jan Vittek; jan.vittek@fel.uniza.sk
}

Received 14 February 2013; Revised 31 July 2013; Accepted 3 August 2013

Academic Editor: Yuqiang Wu

Copyright (C) 2013 J. Vittek and S. Ryvkin. This is an open access article distributed under the Creative Commons Attribution License, which permits unrestricted use, distribution, and reproduction in any medium, provided the original work is properly cited.

A decomposed sliding mode control of the drive with an interior permanent magnet synchronous motor and flexible coupling is presented. Decomposition exploits principles of vector control to divide motor into channel for control of magnetic flux and channel for control of torque separately. Sliding mode control principles are exploited to keep demanded value of magnetic flux and to control load angle in the presence of vibration modes and external disturbances. To obtain continues voltage as a control variable a smoothing integrator follows signum function in both channels. As a modification the switching governed by signum function is replaced by the high gain including rearrangement of the control system block diagram. The simulations indicate that the control system yields the desired robustness and further investigations are recommended.

\section{Introduction}

Fulfillment of the increasing quality requirements to the performance of many industrial drives nowadays is not possible in the presence of the elastic features of the drive's mechanical parts. Such features can cause torsion vibrations, which decrease the drive dynamic accuracy and in some specific cases can even lead to instability of the overall control system. Product degradation is usually a result of them. The problem of torsional vibration elimination is prevalent not only in high-power application such as rolling-mill drives but also conveyer and cage-host drives. Today due to the progress in power electronic and microprocessor systems that allow controlling the electromagnetic torque of motor almost without any delay and expand a pass-band up to the frequencies comparable to a resonance frequencies of the controlled plant mechanical parts, the problem of torsional vibrations appears also in many medium and low-power applications such as servo-drives, throttle drives, and robot arm drives including space applications [1-6].

It is well known that the classical PI controller is not effective in controlling the plant with elastic joints. Many different control concepts that effectively damped torsional vibrations have been proposed and developed, for example, $[7,8]$. For instance additional feedback from selected state variables of the two-mass system can effectively damp torsional vibrations [4].

In the case, if the control plant parameters can change, the problem is getting more complicated and many advanced control concepts have been proposed as solution. For example, the solution based on the robust control theory exploiting the $H_{\infty}$ is presented in [9]. The genetic algorithm is used to receive the estimates of control plant parameters. The Model Predictive Control is another tool that is used to solve the above-mentioned control problem. The Model Predictive Control generates an optimal control taking directly into account all available and/or estimated information about the control variables, control plant parameters, and, for example, the input and output constraints of the plant [1012]. However, the real-time implementation of such control is currently limited because of need to solve complex optimization problem in the presence of complete information about all control variables and plant parameters. 
Different approaches depend on application of a slidingmode control, robustness of which is capable to eliminate influence of control plant parameters changes and also to eliminate external disturbances if these are presented in the control system dynamics [13-16].

The main goal of this paper is to present the novel sliding mode control based control system for plant with elastics connections that combines robustness with chattering free control while exploiting voltage source inverter (VSI).

1.1. Control Plant Model. The control plant has three important parts:

(i) VSI that feeds IPMSM;

(ii) Interior Permanent Magnet Synchronous Motor;

(iii) Elastic mechanical transmission, for example, a gearbox, with external load.

Mathematical models of these plant parts are necessary for control system design; therefore, their description is presented in the following

1.2. Voltage Source Inverter. As it is known feed voltages corresponding to the demanded operation mode of the synchronous motor should be generated on its windings. A supplyline with three-phase voltages $(A, B, C)$ with fixed amplitude and frequency usually acts as the source of power energy. Power converters are used to transform these voltages into three-phase supply voltages for a synchronous motor $(R, S, T)$ having a variable magnitude and frequency. Threephase power converters are built using power transistors working in relay mode [17].

The phase output voltage of the power converter represents in this case a sequence of voltage impulses. This high frequency sequence is averaged in the synchronous motor winding owing to its filtering property. The theoretical background is based on Shannon-Kotelnikov or the sampling theorem $[18,19]$. This average voltage could be considered, as a continuous phase voltage on the synchronous motor winding needed to solve the control problem. Thus, the phase output voltage of the power converter from the control viewpoint has a dual character:

(a) pulse-by nature;

(b) continuous average-for electric motor control.

To realize the specified transformation of the three-phase voltages widely the scheme of power transformation with a direct current link (Figure 1) is used. In this case the three-phase supply line voltages are rectified by means of a three-phase rectifier and smooth out by an output capacitor. Obtained constant voltage supplies an input of the threephase VSI. From DC link voltage inverter produces desired three-phase voltages of variable frequency and magnitude.

The voltage source inverter [16] transforms a DC input voltage in three-phase variable voltages of a constant or variable frequency and/or amplitude. To obtain the needed phase voltage, the voltage transformation pulse method is used. It is based on the application of a switch operation mode

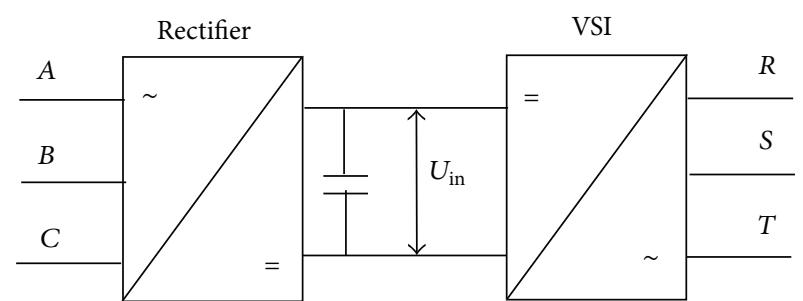

Figure 1: Power converter with a direct current link.

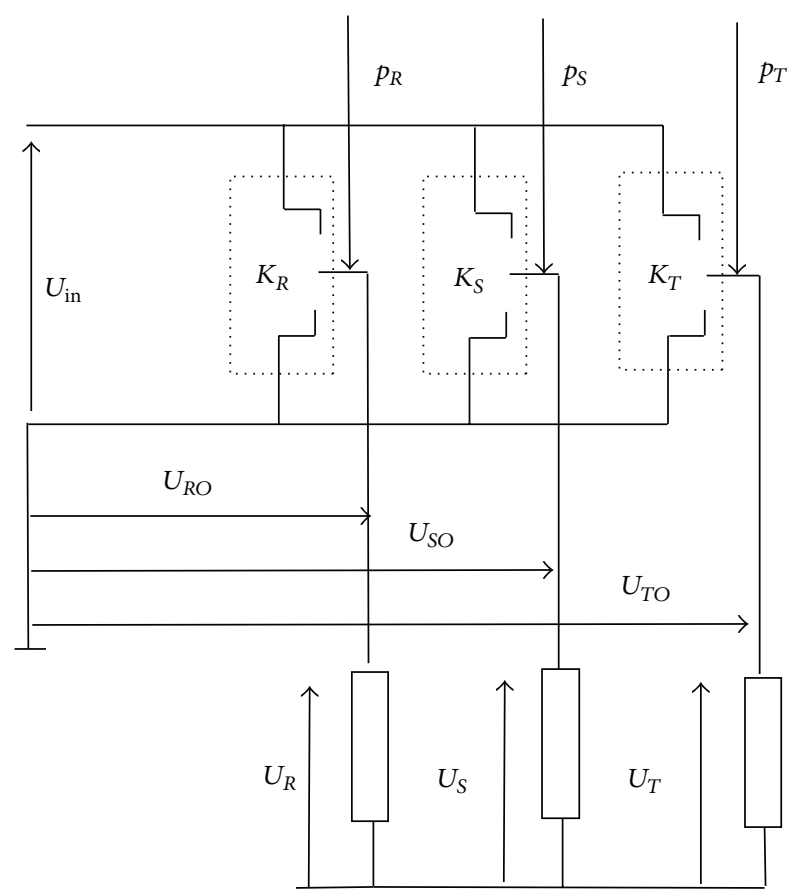

FIgURE 2: The simplified schema of the three-phase VSI bridge.

of power semiconductor devices (switches). They connect any output phase of the load to a source of constant voltage $U_{\text {in }}$.

One of the mostly used VSI is the three-phase bridge with the isolated neutral, shown in Figure 2. It has a star connected load with a parallel connection of three phases on-off power switches $K_{j}(j=R, S, T)$. A control signal $p_{j}\left(p_{j} \in\{0,1\}\right)$ of each switch controls the connection of the VSI output phase load either to a positive potential or to the negative one of the constant input voltage $U_{\text {in }}$.

Thus, depending on a control signal of each switch the output phase voltage $U_{j 0}$ is equal at any moment to input constant voltage $U_{\text {in }}$ or zero. The vector of VSI output voltage $\mathbf{U}^{T}=\left(U_{\alpha}, U_{\beta}\right)$, in a fixed orthogonal coordinate system $(\alpha, \beta)$, is defined by Clark transformation as

$$
\left|\begin{array}{l}
U_{\alpha} \\
U_{\beta}
\end{array}\right|=\frac{2}{3}\left|\begin{array}{ccc}
1 & -\frac{1}{2} & -\frac{1}{2} \\
0 & \frac{\sqrt{3}}{2} & -\frac{\sqrt{3}}{2}
\end{array}\right| \begin{gathered}
U_{R O} \\
U_{S O} \\
U_{T O}
\end{gathered} \mid
$$

where numerical factors of a transformation matrix are directing the phase load terminals $(R, S, T)$. As phase voltages have relay character the voltage output vector $\mathbf{U}$ can accept 


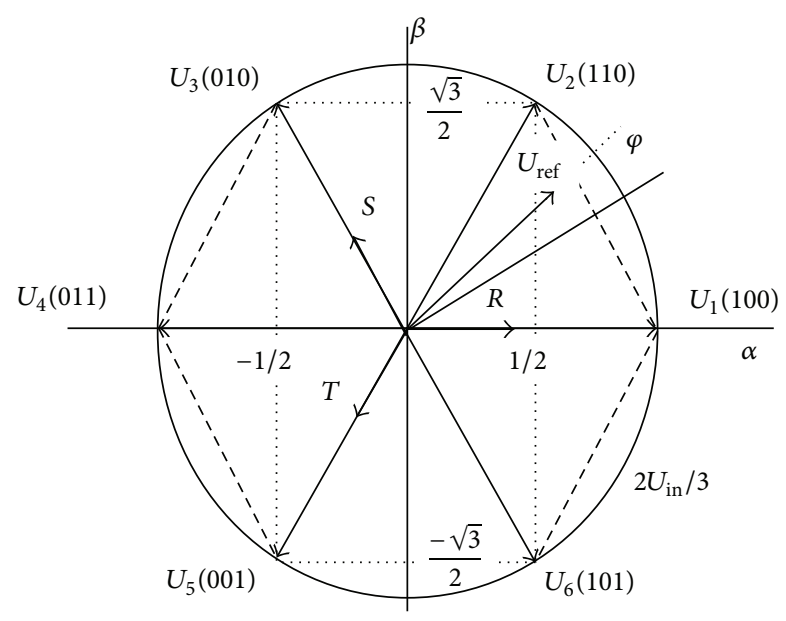

FIGURE 3: Vectors of VSI in complex plane.

only eighth values: two of them are zero $U_{0}$ and other six $U_{i}(i=1, \ldots, 6)$ are nonzero and create the tops of the regular hexagon (Figure 3 ). Its symmetry axes are directed on the phase load terminals.

The module of the above-mentioned six nonzero vectors depends on the load connection and on the value of the input dc line voltage $U_{\text {in. }}$. In the load, connected as "star" circuit, it is equal to $2 U_{\text {in }} / 3$. The combination of phase switches $\left(p_{R}, p_{S}, p_{T}\right)$ controls the possible vector of the output voltage $U_{i}$ presented in Figure 3 . The zero vectors correspond to two combinations of the switch positions: either all are connected to positive potential (111) or to negative potential (000).

The VSI switch control, providing the needed output voltage, has two independent parts [20,21]:

(i) the modulation law that defines the part of the modulation period, in which the power switch is connected either to the positive potential $\left(U_{\text {in }}\right)$ or the negative one (0);

(ii) the switching law that defines a sequence of switching of phase switches on the modulation period.

In this case, each phase output voltage represents a sequence of various duration squared impulses, magnitude of which is equal to $U_{\text {in }}$, that is, the feed voltage. A sequence of these impulses, being averaged owing to filtering properties of the load, forms a phase output voltage on the load creating a control tool. In analysis of the VSI operation and control system synthesis, it is therefore necessary to consider the dual character of an output voltage vector. On one hand, it is characterized at any moment by the instantaneous value, caused by instantaneous positions of the power switches. On the other hand, owing to that the averaging properties of the load it possesses an average on the modulation period value defining, the VSI feature is a part of an automatic control system. These problems will be also considered further during the design of control system for the drive employing synchronous motor.

1.3. Interior Permanent Magnet Synchronous Motor. The classical mathematical model that considers the basic physical features of the processes inside of the motor, shown in Figure 4, received by standard assumptions is used for motor description [13-15]. In the frame of these assumptions the mathematical description of IPMSM includes the following three groups of equations:

(i) equations of electric balance in its windings;

(ii) equation of the electromagnetic torque developed by the electric motor;

(iii) equation of mechanical movement (Newton's second law for a rotary motion).

The third equation, based on formula of mechanical movement, is general for all electric motors and has the following form:

$$
J \frac{d \Omega}{d t}=M_{\mathrm{el}}-M
$$

where $J$ is the moment of inertia of all rotational parts reduced to the shaft of rotor, $\Omega$ is the rotor angular speed, $t$ is the current time, and $M_{\mathrm{el}}$ is the electromagnetic torque developed by the motor. $M=\sum M_{l}\left(F_{i}\right)$ is the sum of the torques of the external forces enclosed to a rotor.

The equations of electric balance based on Kirchhoff's second law and the equations of electromagnetic torque are defined by the electric and magnetic circuits of the IPMSM and the physical processes inside of it. In the case of IPMSM the magnetic flux is created by permanent magnets located inside of the rotor. Such placement of permanent magnets gives to the rotor feature of the salient poles and the IPMSM has a nonuniform air gap. Due to nonuniformity of the air gap the magnetic flux creates an additional (reluctance) torque.

It is well known that mathematical description of the electromechanical transformation of power in the IPMSM using the actual phase currents and voltages as independent variables is capable to describe the physical processes inside of the motor. On the other hand such description is relatively complicated for analysis of the dynamical processes and results in a system of nonlinear differential equations with periodically changing coefficients due to change of rotor position.

The successful choice of transformation into axes $(0, d, q)$ plays a fundamental role in the theory of synchronous machines and the theory of automatic drives and clearly proves the following statements. First, thanks to the rotating coordinates system connected to a rotor, the differential equations describing the synchronous motor dynamics have constant coefficients.

Second, orientation of a datum line 0 on the axis of rotation makes the differential equation independent regarding to a zero sequence current. It must be emphasized that the zero sequence current does not participate in the creation of air gap magnetic field and it has no influence on the electromechanical processes in the motor. It creates only an additional loading of windings and semiconductor devices. The traditional connection of motor phase windings "star" or "delta" automatically provides cancellation of a zero sequence current; that is, it eliminates additional thermal losses. It leads 


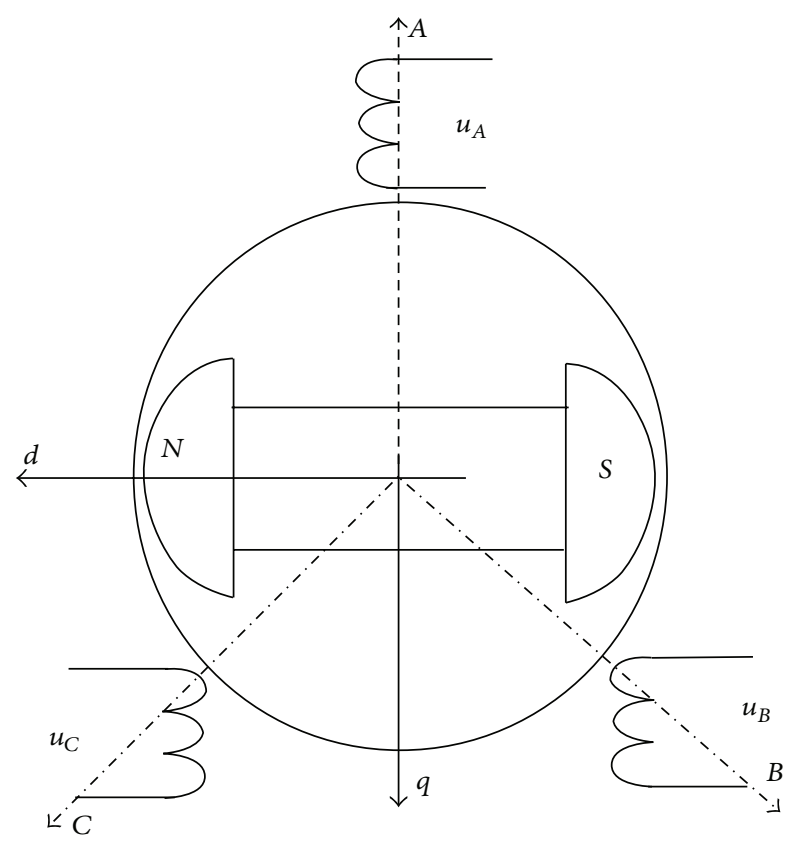

FIGURE 4: IPMSM.

to simplification of mathematical description of synchronous motor. Elimination of differential equation for the zero sequence current reduces the system order. In this case the considered three-phase synchronous motor is replaced by a two-phase "idealized" one (the generalized electric machine), which from the point of view of the electromagnetic processes represents the collector machine.

The transformation from the three-phase coordinates system to the rotating two-phase system $(0, d, q)$ is implemented via a Park transformation matrix:

$$
C=\sqrt{\frac{2}{3}}\left(\begin{array}{ccc}
\cos \gamma_{A} & \cos \gamma_{B} & \cos \gamma_{C} \\
-\sin \gamma_{A} & -\sin \gamma_{B} & -\sin \gamma_{C}
\end{array}\right),
$$

where $\gamma_{j}$ is an electric angle between a rotor axis $d$ and a stator phase axis $j(j=A, B, C)$ and $\sqrt{2 / 3}$ is a factor for power invariance.

The electric variables in a rotating system $i_{d}, i_{q}, u_{d}, u_{q}$ are also connected to phase variables:

$$
I=C I^{\prime}, \quad U=C U^{\prime},
$$

where $I^{T}=\left(i_{d}, i_{q}\right), I^{\prime T}=\left(i_{A}, i_{B}, i_{C}\right), U^{T}=\left(u_{d}, u_{q}\right), U^{\prime T}=$ $\left(u_{A}, u_{B}, u_{C}\right)$.

The equations of electric balance and the electromagnetic moment for IPMSM in the rotating frame are as follows:

$$
\begin{gathered}
\frac{d i_{d}}{d t}=\frac{1}{L_{d}}\left(-r i_{d}+L_{q} \omega i_{q}\right)+\frac{1}{L_{d}} u_{d}, \\
\frac{d i_{q}}{d t}=\frac{1}{L_{q}}\left(-r i_{q}-L_{d} \omega i_{d}-\Psi_{f} \omega\right)+\frac{1}{L_{q}} u_{q}, \\
M_{\mathrm{el}}=\frac{3}{2} p\left[\Psi_{f}+\left(L_{d}-L_{q}\right) i_{d}\right] i_{q},
\end{gathered}
$$

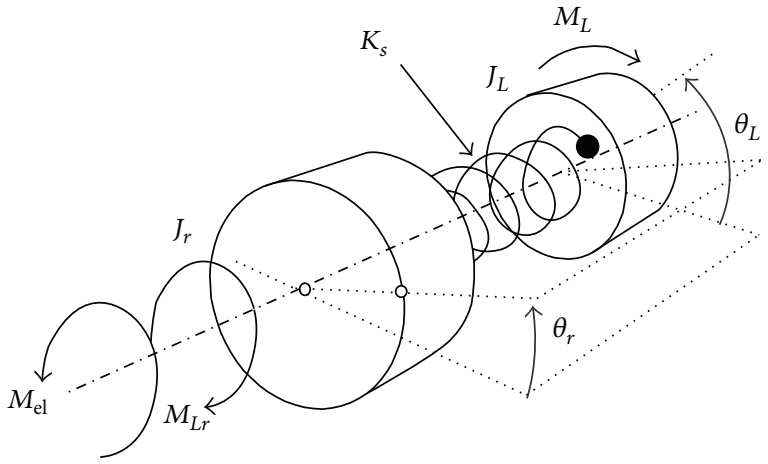

Figure 5: Elastics mechanical transmission.

where $i_{d}, i_{q}$ are the currents in the stator windings; $u_{d}, u_{q}$ are the voltages in the stator windings; $r$ is the active resistance of a stator windings, $L_{d}, L_{q}$ are the direct and quadrature axis inductances of stator windings; $p$ is the number of pair poles; $\omega$ is the electric angular speed; $\Psi_{f}$ is an excitation flux.

1.4. Elastic Mechanical Transmission. The behavior of the drive's mechanical part (Figure 5) causing torsional vibrations could be described as follows. The IPMSM with moment of inertia $J_{r}$ produces the electromagnetic torque on its shaft $M_{\mathrm{el}}$. The angular speed of the motor shaft is equal to $\Omega$. The reduction factor of mechanical train from a motor shaft to the final control element (load) is equal to 1. Load inertia (the moment of inertia $J_{L}$ ) is coupled to the motor via the elastic mechanical connection with a string factor $K_{S}$. Two external disturbance torques $M_{L r}$ and $M_{L}$ operate, respectively, on the rotor and on the load sides. The inertial load rotates with the angular speed $\Omega_{L}$. The angular position of the final control element is $\theta_{L}$.

Based on torque balance the behavior of the elastics mechanical transmission is described by following difference equations:

$$
\begin{gathered}
\frac{d \theta_{r}}{d t}=\Omega_{r}, \\
\frac{d \Omega_{r}}{d t}=\frac{1}{J_{r}}\left[M_{1 l}-M_{L r}+K_{S}\left(\theta_{L}-\theta_{r}\right)\right], \\
\frac{d \theta_{L}}{d t}=\Omega_{L}, \\
\frac{d \Omega_{L}}{d t}=\frac{1}{J_{L}}\left[K_{S}\left(\theta_{r}-\theta_{L}\right)-M_{L}\right] .
\end{gathered}
$$

1.5. Sliding Mode Control Backgrounds. Sliding mode is a special type of behavior of a relay system $[15,16]$ :

$$
\begin{gathered}
\frac{d x(t)}{d t}=l(x, t)+B(x, t) u(t), \\
u_{i}(x, t)= \begin{cases}u_{i}^{+}(x, t) & \text { if } S_{i}(x, t)>0 \\
u_{i}^{-}(x, t) & \text { if } S_{i}(x, t)<0,\end{cases}
\end{gathered}
$$


where $x(t)$ is a state vector, $x(t) \in R^{n} ; l(x, t)$ is a system vector, $l(x, t) \in R^{n} ; u(t)$ is a control vector, $u(t) \in R^{m}, n \geq m ; u_{i}(t)$ is a component of the control vector $u(t), i=\overline{1, m} ; B(x, t)$ is a matrix, $B(x, t) \in R^{n \times m} ; S_{i}(t)$ is a switching function.

The main feature of this mode is that none of the used general switched structures can realize such behavior. The sliding mode occurs in the intersection of all m surfaces

$$
f_{i}=0
$$

by high frequency switching of the control components $u_{i}(x, t)$ (8). The vector function $F^{T}=\left(f_{1}, \ldots, f_{m}\right)$ is a function of the system variables $x(t)$. Usually it is an error function that must be led to zero by using the vector switching function $S^{T}=\left(S_{1}, \ldots, S_{m}\right)$.

Formally, the aim of control is to force state system, $x(t)$ to reach the manifold

$$
F=0
$$

and "slides" on this manifold to the reference point, independently of the system dynamics.

In this case the control design procedure is decoupled into the two tasks:

(i) sliding mode design in the space of the vector function $F \in R^{m}$;

(ii) motion design on the intersection of all $m$ surfaces in the state space with order $(n-m)$.

Solution of the first task is based as it is considered in sliding mode analysis on the Lyapunov stability of the control plant in the space of the vector function $F \in R^{m}$. A typical sliding mode control variable, $u$, has the form

$$
u=-U(x) \operatorname{sgn}(F)
$$

where $U(x)$ is the square diagonal matrix of control magnitude and $\operatorname{sgn}(F)$ is the vector of the signs of error functions, $[\operatorname{sgn}(F)]^{T}=\left(\operatorname{sgn} f_{1}, \ldots, \operatorname{sgn} f_{m}\right)$. It guarantees that the system state will reach the sliding manifold in finite time from the initial condition, which has been bounded by the value of the matrix $U(x)$ constituent and will keep to it. This magnitude bounds the uncertainty of the system, the load value unto which the system is commonly robust. Special task is the design of a transition law between the designed control (11) and the switching control (8), which depends on the features of the relay system (7).

Motion on the sliding manifold is described by the equivalent control $u_{\mathrm{eq}}(x)$. Its calculation is based on the condition that the time-derivative of the function $F$ on the system trajectories is equal to zero

$$
\frac{d F}{d t}=G l+G B u_{\mathrm{eq}}=0
$$

where $G(x)=\{\partial F / \partial x\}, G(x) \in R^{m \times n}$ is a gradient matrix; $\operatorname{det} G B \neq 0$.

In this case, the equivalent control, $u_{\mathrm{eq}}(x)$ that is a continuous control which guarantees the same motion if all needed information about the load and the system uncertainty were available, is calculated

$$
u_{\mathrm{eq}}=(G B)^{-1} G l \text {, }
$$

and the system motion in the sliding mode is described as

$$
\frac{d x}{d t}=l-B(G B)^{-1} G l .
$$

Using (10) the system order can be reduced to $(n-m)$, and the system description is

$$
\frac{d x_{1}}{d t}=l_{1}\left[\left(x_{1}, t\right)\right]
$$

where $x^{T}=\left(x_{1}, x_{2}\right), x_{1} \in R^{n-m}, x_{2} \in R^{m} ; l_{1}\left[\left(x_{1}, t\right)\right], l_{1} \in$ $R^{n-m}$.

\section{Sliding Mode Control Design}

2.1. Control Goal. An electrical motor in the drive structure carries out a transformation of the electric energy into a mechanical one that moves the mechanisms participating in the working process. Technology requirements for this process define the necessity and expediency of maintenance at the reference level of those or other mechanical variables, for example, position, speed, acceleration, torque, and so forth, of the mechanism tip.

In our case the main adjustable variable is a load position $\theta_{L}$ connected to the rotor shaft by elastics connection. This position should be equal to the reference values $\theta_{L z}(t)$. In the most general case the reference value $\theta_{L z}(t)$ is a function of time. This is essential for the servo control problem. The actual load angular position should reproduce all changes of the reference value with prescribed accuracy. Special cases of this problem are

(i) stabilization of the load position at the reference constant level;

(ii) the load angular position change under the reference law;

(iii) restriction of the load angular position by an admissible value.

The number of the control variables depends on the number of the control plant independent controls. In the case of IPMSM there are two independent controls (see (4)) and there is a possibility together with the requirements on the mechanical coordinate to fulfill the requirement on profitability of drive work. Its indicators are the efficiency coefficient, characterizing power losses, and $\cos \varphi$, characterizing consumption of the reactive power.

The main control goal is to maintain the reference value of the load angular position $\theta_{L}$ in combination with power requirements. In relation to the reference demands, any change of the load torque has to be carried out exactly and fast. Due to discontinuous work of power converter solution of control problem requires formation of such switch control to comply with the above-mentioned requirements. Referring 
to the theory of systems with discontinuous controls in this case it is quite natural. Essential nonlinearities of the drives supplied by power converter can bring up a variety of approaches to the solution of a control design problem.

Several basic approaches to the synthesis of IPMSM drives control can be defined:

(i) single-loop control;

(ii) single-loop decomposition value reference;

(iii) cascade (subordinated) control.

In the first case the drive is considered a unit. The control synthesis interfaces to the solution of the nonlinear problem. The switching frequency and duration of power switches on states are generated automatically in the closed loop, as an auxiliary element in the solution of the primary drive control goal. Such systems possess high dynamics and small sensitivity to drive parameter changes and to external disturbances. Unfortunately, the automatically generated switching frequency of the power switches is not constant because it depends on the initial conditions. This leads to the power switches losses increase and to the drive mechanical noise.

In the second case two independent problems are considered: a problem of IPMSM control design and a problem of power converter control design. After transformation of IPMSM into system rotating at rotor speed the control law synthesis is based on the assumption that the power converter generates DC input currents and voltages responding to the solution of the primary control goal. Inverse Clark and Park transformations change the constant input currents and voltages into voltages and currents with variable frequency and magnitude. The problem of the power converter control is to select suitable switching of eight available switching vectors to follow required voltages with variable magnitude and frequency as close as possible providing the complete solution of the control problem.

Power converter control deals with the use of the modulation techniques based on a high frequency to generate the input voltages of the motor. DC output currents and voltages of control algorithm represent for the power converter a sequence of impulses of various durations. This sequence of impulses, being averaged due to the filtering effect of the load, that is, the IPMSM, forms a continuous load voltage, which is subject to control. In this case the indicators characterizing the discontinuous control, that is, its modulation such as switching frequency and pulse ratio are formed outside of power converter; that is, feed forward (program) control is executed. However, with such type of control, the automatic compensation of external disturbances and internal parameter changes is possible only in the upper loop of the mechanical coordinate control.

The control problem, in the third case, exploits the principles of the cascade (subordinated) control. Decomposition of an initial problem of the drive control on drive processes rates is required. The control problem for each of processes is handled separately. Natural splitting of the drive processes respects: the electromagnetic modes, which are fast and the mechanical modes, which are usually slow. In relation to the fast processes, the mechanical variables are quasiconstants.
Hence, the electromagnetic processes control problem is reduced to a control of the electromagnetic torque. The torque reference is formed in a slow control loop of the mechanical movement, which is based on the movement equation (2). Since the value of the electromagnetic torque is defined by the DC values (as the current in the DC motor winding), the control problem for a fast internal loop is reduced to a feedback current control using the power converter. Change of discontinuous controls, that is, the switching frequency and duration of the switching of power switches are generated automatically in the closed loop, as an auxiliary element of the control problem solution. The current control loop possesses high dynamics and small sensitivity to motor parameter changes and external disturbances. However, it is necessary to notice that, as in the first case, the switching frequency of power switches is generated automatically and its value depends on initial conditions. Therefore, it similarly increases switching losses of the power converter switches and increases mechanical drive noise.

\section{Single-Loop Control}

3.1. Sliding Mode Functions Selection. As it was explained above the basic controlled variable in an electrical drive is the load angular position $\theta_{L}$, which ideally should be equal to the reference $\theta_{L z}(t)$. There are two different operating conditions for the IPMSM. Better electrical drive performance can be achieved by obtaining the largest possible efficiency while keeping the power factor equal to one. For simplification of the control design the above-mentioned power requirements could be transformed into a reference of stator current component, $i_{d z}$ computed for the IPMSM known parameters and a measured component of the stator current $i_{q}$. In practice, to simplify control algorithm and to keep linkage flux of motor constant $[22,23]$, the reference of the stator current component $i_{d}$ is prescribed as $i_{d z}=0$. In this case value $\cos \varphi$ is near its optimum values [16].

Using such references, that is, the load angular position $\theta_{L z}(t)$ and the stator current $i_{d z}(t)$, it is possible to achieve specific requirements of the IPMSM control. Having chosen those references, the electrical drive behavior is described by the error functions of the controlled variables, which are deviations of the controlled variables actual values and the references:

$$
\begin{aligned}
Z_{1}= & \frac{d^{4}\left(\theta_{L z}-\theta\right)}{d t^{4}}+C_{3} \frac{d^{3}\left(\theta_{L z}-\theta\right)}{d t^{3}} \\
& +C_{2} \frac{d^{2}\left(\theta_{L z}-\theta\right)}{d t^{2}}+C_{1} \frac{d\left(\theta_{L z}-\theta\right)}{d t} \\
& +C_{0}\left(\theta_{L z}-\theta\right) \\
Z_{2}= & i_{d z}-i_{d},
\end{aligned}
$$

where $C_{0}, C_{1}, C_{2}, C_{3}$ are constants.

The order of the error function depends on the model of control plant. Its derivation must be linear function of 
the control variables. To solve this control problem, for example, to make the error functions equal to zero, it means to synthesize switching control actions of power converter output voltages, which feeds the IPMSM stator windings. From the point of view of output voltages the above error function (16) would be zero simultaneously.

In this case, current, $i_{d}$ is equal to its reference value, $i_{d z}$, and the error between the reference, $\theta_{L z}(t)$, and the actual load angular position, $\theta_{L}$, will tend to zero if the factors of difference equation have been chosen from a condition of a desired placing of difference equation roots on the left half of complex plane. The convergence rate of actual value to the reference one is defined by the positions of the roots.

One of the possible variants to maintain the simultaneous equality for functions (16) as zero is the organization of a sliding mode on crossing of surfaces $Z_{1}=0$ and $Z_{2}=$ 0 . The solution for this problem is based on the two-step design procedure [17]. The initial problem of control design is divided into tasks of smaller complexity, as a two-step process, taking into account operating features of the IPMSM and power converter. As the first step, features of the motor are considered only. A problem can be solved by synthesis of a sliding motion using a fictitious control vector and a classical mathematical model of the IPMSM (see Park equations (3)). The fictitious control variables are two-phase voltages of the bi-phase Park model. They do not exist as real; however, such model is very convenient for the control system design. The real motor for control is a three-phase voltage system formed by the converter phase switching control. The second step therefore carries out the transition to actual controls taking into account functioning features and peculiarities of the three-phase semiconductor power converter of various types.

3.1.1. The First Step. As it was mentioned the possibility to organize sliding movement and to design the necessary control variables can be solved using the equation for initial dynamic system motion projection, for example, the IPMSM, at an error subspace of the controlled variables $Z$. If Park description of the IPMSM and the control variables specified above are used, the projection equation has the following form:

$$
\frac{d Z}{d t}=F+A U
$$

where vector $F=d z_{z} / d t-G f$ and matrix $A=G B$ do not depend on the control vector $U$ but are defined by the column vector $F^{T}(x, t)=\left(f_{1}, f_{2}\right)$ and the control matrix $B(x, t)$ of the motor, respectively. The existing conditions directly depend on matrix $A$.

Motor and load can be modeled by the system of differential equations of seven order (5), (6), where $\mathbf{U}^{T}=$ $\left(u_{q}, u_{d}\right)$ is a fictitious control vector, components of which are voltages, $u_{d}, u_{q}$ on a fictitious bi-phase windings in a rotating coordinate system $(d, q)$. The error function vector $Z^{T}=\left(Z_{1}, Z_{2}\right)$ contains only two components. In this case, vector $F$ and matrix $A$ can be combined into equation for matrix $A$ :

$$
A=\left(\begin{array}{cc}
-\frac{K_{S}}{J_{L} J_{r}}\left(\frac{L_{d}-L_{q}}{L_{d}}\right) i_{q}-\frac{K_{S}}{J_{L} J_{r}}\left[\left(\frac{L_{d}-L_{q}}{L_{q}}\right) i_{d}+\Psi_{f}\right] \\
0 \\
-\frac{1}{L_{d}}
\end{array}\right)
$$

After inspection of matrix $A$, it is visible that component $u_{q}$ of a control vector $U$ has not influence on sliding mode occurrence at the surface $Z_{2}=0$. Hence, using a method of control hierarchy $[15,16]$, it is possible to carry out decomposition of an initial design problem into two independent onedimensional problems on the existence of one-dimensional sliding modes on surfaces $Z_{1}=0$ and $Z_{2}=0$. The control design and a choice of the control variables magnitudes for each of them are carried out under assumption for existence conditions of one-dimensional sliding movement. The sliding motion is designed at first on surface $Z_{2}=0$ using $u_{d}$ and then on surface $Z_{1}=0$ using control $u_{q}$.

The control algorithm

$$
u_{d}=u_{d 0} \operatorname{sgn} Z_{2}
$$

and the inequality

$$
u_{d 0} \geq\left|u_{d \text { eq }}\right|=\left|f_{2}\right|
$$

provide contrast of signs on error function $Z_{2}$ and its derivative, that is, the sliding mode performance for the above specified existence conditions for a scalar case.

By solving the control design problem for the second component of a control vector $u_{q}$, the principle of control hierarchy is used. It is supposed that sliding mode on surface $Z_{2}=0$ takes place, and it is necessary to provide sliding mode on surface $Z_{1}=0$. In this case, according to a method of equivalent control (14), in the first equation of system (18) instead of control $u_{d}$, its value, namely, the equivalent control, $u_{d \text { eq }}$, is substituted. Sliding mode on surface $Z_{1}=0$ and, hence, on the crossings of surfaces $Z_{1}=0$ and $Z_{2}=0$ will take place, if

$$
\begin{gathered}
u_{q}=u_{q 0} \operatorname{sgn} Z_{1}, \\
u_{q 0} \geq\left|u_{q \text { eq }}\right|=\left|f_{1}\right| .
\end{gathered}
$$

It is important to notice that on "reasonable" operating modes the magnetic flux $\Psi=\Psi_{f}+\left(L_{d}-L_{q}\right) i_{d z}$ causing electromagnetic torque $M_{\mathrm{el}}$ has to be different to zero; otherwise there would be no possibility to control the electromagnetic torque of the synchronous motor. Hence, at $\Psi \neq 0$ there is a basic possibility of performance of condition (22) at the expense of a choice of an enough large value, $u_{q 0}$, that is, maintenance of sliding mode existence along the surface $Z_{1}=$ 0 .

As a first stage result, decomposition of an initial design problem was carried out and two one-dimensional problems about the occurrence of sliding modes were considered independently. The received equations (19), (21) describe 
working control, and the inequalities (20), (22) at $Z=0$ define a class of admissible perturbations and the references reproduced without a dynamic tracking error.

3.1.2. The Second Step. It is a transition from the fictitiously entered control vector, $\mathbf{U}^{T}=\left(u_{d}, u_{q}\right)$ to the real control of power converter feeding the IPMSM. The stator windings are fed by a three-phase VSI. It can accept only 8 output voltage vectors, six of them having nonzero values, separated an angular distance of $\pi / 3$ from each other and two of them with zero value (Figure 3 ).

Each of these vectors has a fixed direction, and none of them coincides with the formally entered control vector, $\mathbf{U}^{T}=$ $\left(u_{d}, u_{q}\right)$ either in direction or in value. Values of magnitude of the components formally entered control vector are chosen according to inequalities valid for the control variables in the rotating coordinate system $(d, q)$.

For the output phase voltages of the power converter design it is possible to use (4) with a transformation matrix (3), connecting formally entered control variables $u_{d}, u_{q}$ and real phase voltage. In this case the phase voltages obtained by the sliding mode designed at the first step will represent sinusoidal ones with frequency equal to multiple angular speed of the IPMSM rotor and both of variable magnitude and phase. These phase voltages including a high-frequency component could be obtained on the output of power converter by using high-frequency PWM, which requires calculation of the time length for switches on state and corresponding sequence of switching. However, such approach requires additional calculations in connection with the allocation of equivalent control variables (necessary for PWM calculation) and will have negative influence on one of the basic property of sliding mode, which is the simplicity of implementation.

The alternative approach to the design of power converter output phase voltages is based on a sufficient existence of a sliding mode condition in the systems with redundant control [16]. Conditions for choices of magnitude values of formally entered control variables are based on inequalities. It allows space allocation of fictitious control variables, in this case $u_{d}, u_{q}$, in the area of the admissible control variables $U^{*}$ guaranteeing sliding mode existence. For the design of real control variables, for example, three phase voltages, it is obvious that their total projections to axes $(d, q)$ coincided on a sign with the designed fictitious controls and values of projections satisfied sliding mode inequalities on crossing of chosen surfaces through the projections value, that is, the values of the formally entered control variables $u_{d}, u_{q}$, will change during the motor operation.

For the design of such control variables it is necessary to transform the domain of admissible controls received in the rotating coordinate system using an inverse linear Park transformation:

$$
\left[\begin{array}{l}
u_{\alpha} \\
u_{\beta}
\end{array}\right]=\left[\begin{array}{cc}
\cos \gamma_{A} & -\sin \gamma_{A} \\
\sin \gamma_{A} & \cos \gamma_{A}
\end{array}\right] \cdot\left[\begin{array}{l}
u_{d} \\
u_{q}
\end{array}\right]
$$

at a fixed coordinate system of the control space $u_{d}, u_{q}$.

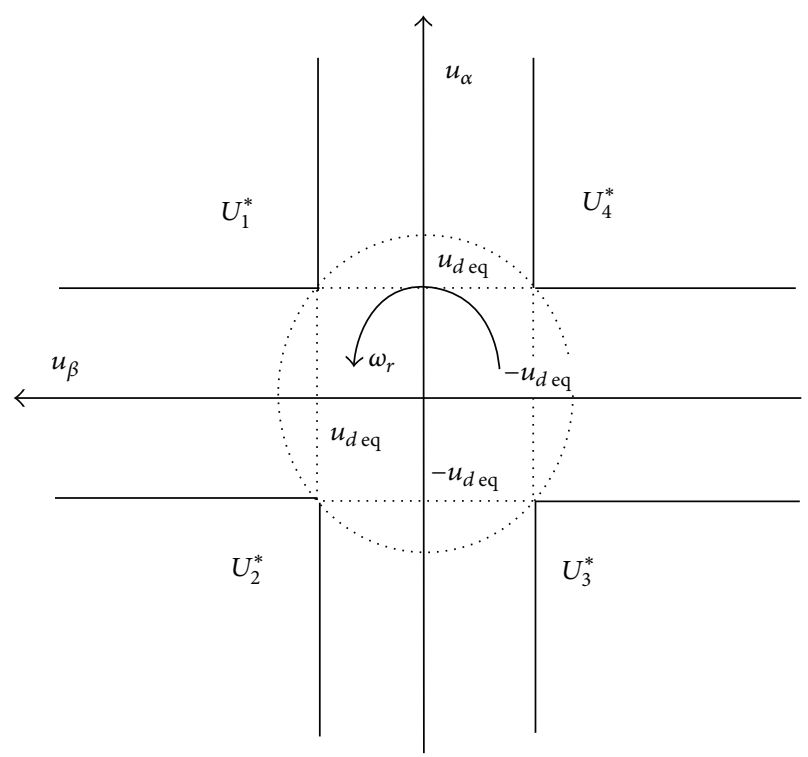

FIGURE 6: Sliding mode areas for admissible control $U^{*}$ in stationary coordinate system $\left(u_{\alpha}, u_{\beta}\right)$.

In this fixed coordinate system the relative position of this area and the output vectors of the power converter (23) must be analyzed again. The transformed area of admissible controls $U^{*}$ (Figure 6) has the same configuration as the original area in the rotating coordinate system $\left(u_{d}, u_{q}\right)$, but it is not fixed. Instead, it rotates with the electrical rotor speed $\omega$.

VSI output voltage vector can accept six nonzero values, separated an angular distance of $\pi / 3$ from each other (Figure 3). After direct check there is no doubt that at all the values $\gamma_{j}(j=R, S, T)$ and any values of $\operatorname{sgn} Z_{1}$ and $\operatorname{sgn} Z_{2}$ of each of the subareas of area of admissible values of control will belong to at least one of six realized nonzero values of a VSI output voltage vector, if

$$
\begin{gathered}
U_{\text {in }} \geq U_{\text {in }}^{*}=\frac{3}{2} \sqrt{u_{d 0}^{2}+u_{q 0}^{2}+\sqrt{3} u_{d 0} u_{q 0}}, \\
\operatorname{sgn} S_{j}=\operatorname{sgn}\left(k \operatorname{sgn} S_{2} \cos \gamma_{j}-\operatorname{sgn} S_{1} \sin \gamma_{j}\right), \\
\sqrt{\frac{8 U_{\text {in }}^{2}+9 u_{d 0}^{2}+3 \sqrt{3} u_{d 0} \sqrt{16 U_{\text {in }}^{2}-9 u_{d 0}^{2}}}{24 U_{\text {in }}^{2}-9 u_{d 0}^{2}-3 \sqrt{3} u_{d 0} \sqrt{16 U_{\text {in }}^{2}-9 u_{d 0}^{2}}}} \\
\leq k \leq \sqrt{\frac{24 U_{\text {in }}^{2}-9 u_{q 0}^{2}-3 \sqrt{3} u_{q 0} \sqrt{16 U_{\text {in }}^{2}-9 u_{q 0}^{2}}}{8 U_{\text {in }}^{2}+9 u_{q 0}^{2}+3 \sqrt{3} u_{q 0} \sqrt{16 U_{\text {in }}^{2}-9 u_{q 0}^{2}}}} .
\end{gathered}
$$

It is obvious that in this case there is a possibility to allocate ranges of angle values $\gamma_{j}$, at which the sign of the VSI phase switch control $p_{j}$ coincides with the sign or the opposite one of the fictitious control sign $Z_{1}$ or sign $Z_{2}$ and does not depend on a sign of the second one. Angle values of $\gamma_{j}$, at which a sign change of the operating signal occur and values of control signal, $p_{j}$ are defined by the formula (25). 
In connection with the above, the control law of switching variables formation, $p_{j}$, which depends on angle values, $\gamma_{R}$ can be presented in the form of a logic table with two input variables: an angle rotor position and four signs functions.

The obtained conditions of sliding mode existence are sufficient for any instantaneous values $u_{d 0}, u_{q 0}$ taken from the sets caused by inequalities of a choice of control magnitudes, that is, for sliding movement on both surfaces $Z_{1}=$ 0 and $Z_{2}=0$. It is necessary to notice that the offered sliding mode control is robust and has not high requirements besides constancy of input voltage VSI $U_{\text {in }}$ and definition of rotor angular position $\gamma_{R}$. The sliding mode will remain in operation even if the value of input voltage VSI, $U_{\text {in }}$ changes substantially. The unique limitation according to inequality (24) is the bottom bound of its change $U_{\text {in }}^{*}$. This way it is possible to simplify the scheme of a direct current link and to refuse the smoothing capacity. The angular position should be defined completely within one of 12 sectors. According to (24) and (26) from which $U_{\text {in }}>U_{\text {in }}^{*}$ high demands also are not made to border definition between sectors on accuracy.

3.2. Single-Loop Decomposition Control. As it was written above the main disadvantage of the single-loop sliding mode control is that the automatically generated switching frequency of the power switches is not controllable. It depends on the initial conditions and could vary in a wide range. This leads to the negative effects as increase of power switches losses and to higher drive's mechanical noise.

One of the possible ways to eliminate this disadvantage is exploitation of the single-loop decomposition control considering separately two independent problems: a problem of IPMSM control design and a problem of power converter control design. The power converter control problem deals with the exploitation of the modulation based on a highfrequency connection to the input voltages of motor phase windings independently.

As the first step of a single-loop decomposition control fictitiously entered control voltage vector, $\mathbf{U}^{T}=\left(u_{d}, u_{q}\right)$ is replaced with the voltage derivations $\mathbf{V}^{T}=\left(v_{d}, v_{q}\right)$

$$
\frac{d u_{d}}{d t}=v_{d}, \quad \frac{d u_{q}}{d t}=v_{q}
$$

where $\mathbf{V}^{T}=\left(v_{d}, v_{q}\right)$ is a fictitious control vector.

In this case the IPMSM with the elastics joints is modeled by a nine-order system of differential equation (5), (6), (28). The error function vector $Z_{T}=\left(Z_{1}, Z_{2}\right)$ contains again two components. However, the order of the error functions is 1 degree higher:

$$
\begin{aligned}
Z_{1}= & \frac{d^{5}\left(\theta_{L z}-\theta\right)}{d t^{5}}+C_{4} \frac{d^{4}\left(\theta_{L z}-\theta\right)}{d t^{4}} \\
& +C_{3} \frac{d^{3}\left(\theta_{L z}-\theta\right)}{d t^{3}}+C_{2} \frac{d^{2}\left(\theta_{L z}-\theta\right)}{d t^{2}} \\
& +C_{1} \frac{d\left(\theta_{L z}-\theta\right)}{d t}+C_{0}\left(\theta_{L z}-\theta\right), \\
Z_{2}= & \frac{d\left(i_{d z}-i_{d}\right)}{d t}+K_{0}\left(i_{d z}-i_{d}\right),
\end{aligned}
$$

where $C_{0}, C_{1}, C_{2}, C_{3}, C_{4}, K_{0}$ are constants.
In this case, the error between the reference value $i_{d z}$ and the actual current $i_{d}$ by $K_{0}>0$ tends exponentially to zero according to a law having a time constant equal to $1 / K_{0}$. The error between the reference, $\theta_{L z}$, and the actual load angular position, $\theta_{L}$, will tend toward zero if the factors of difference equation have been chosen from a condition of a desirable placing of difference equation roots on the left half of complexplane. The convergence rate of actual value to the reference one is defined by the positions of the roots.

For the design of the sliding mode on the intersection of the surfaces $Z_{1}=0$ and $Z_{2}=0$ (17) is used. However the vector $F$ and matrix $A$ are received using the complete drive model (5), (6), and (27). As a result, two relay control variables, $v_{d}$, $v_{q}$, defined by (19)-(23) guarantee robustness property in the sliding mode.

For the second step, which is power converter real control design fictitiously entered control vector $\mathbf{V}^{T}=\left(v_{d}, v_{q}\right)$ received in the first step cannot be used. VSI control can change only the average value of the output voltage. The reference voltage value of VSI must be received via integration of the fictitiously entered control vector, $\mathbf{V}^{T}=\left(v_{d}, v_{q}\right)$ components. In this case the indicator characterizing the VSI discontinuous control, that is, its modulation, is formed for the power converter from the outside, that is, feed forward (program) control. The pulse ratio is formed as a function of the reference voltage value.

Due to choice of switching frequency there is a possibility to minimize drive's switching losses. However, it must respect the fact that to obtain good results the PWM frequency must be a few times higher than the band pass highest frequency of the controlled plant.

One disadvantage of this approach if compared with classical one is the need of the additional derivation of the control variables. It is well known that the derivations of high order have larger influence on the control system dynamics. Another approach that allows using the integral of the control variable error instead of the 5 th order derivation was investigated. In this case all advantages of the above decomposed sliding mode control design are saved.

The behavior of the IPMSM with the elastics connection is described by the same seventh-order differential equation system of (5), (6) with the fictitious control vector $\mathbf{U}^{T}=$ $\left(u_{q}, u_{d}\right)$. However, the error functions of the controlled variables (16) have an additional integrate part

$$
\begin{aligned}
Z_{1}= & \frac{d^{4}\left(\theta_{L z}-\theta\right)}{d t^{4}}+C_{3} \frac{d^{3}\left(\theta_{L z}-\theta\right)}{d t^{3}} \\
& +C_{2} \frac{d^{2}\left(\theta_{L z}-\theta\right)}{d t^{2}}+C_{1} \frac{d\left(\theta_{L z}-\theta\right)}{d t} \\
& +C_{0}\left(\theta_{L z}-\theta\right)+C \int\left(\theta_{L z}-\theta\right) d t, \\
Z_{2}= & \left(i_{d z}-i_{d}\right)+K \int\left(i_{d z}-i_{d}\right) d t,
\end{aligned}
$$

where $C, C_{0}, C_{1}, C_{2}, C_{3}, K$ are constants.

For the design of the sliding mode on the intersection of the surfaces $Z_{1}=0$ and $Z_{2}=0$, (17) is used. However, the 


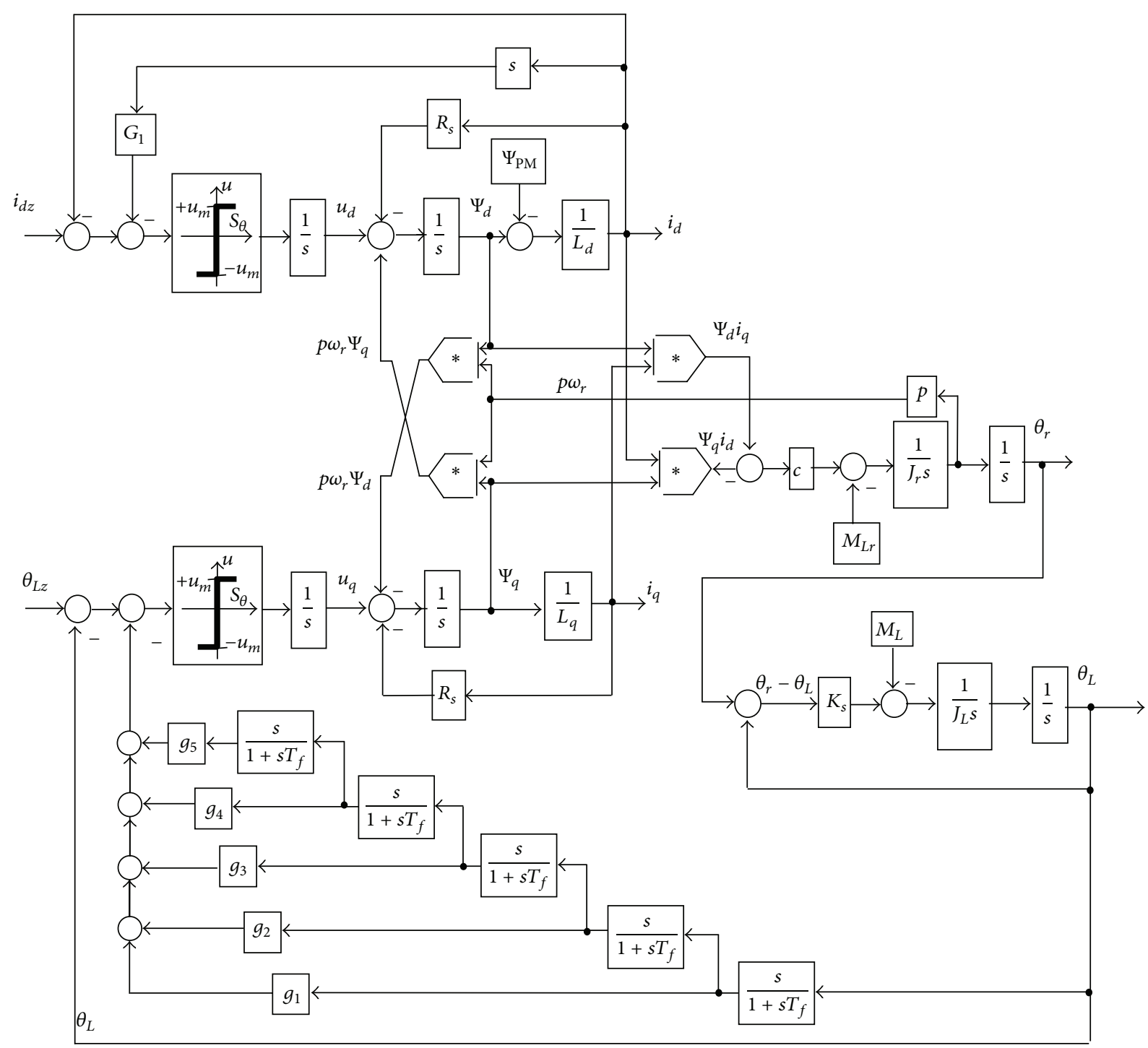

FIGURE 7: Overall block diagram of decomposed SMC system with IPMSM and flexible coupling.

vector $F$ and matrix $A$ are obtained using the model of drive described by (5), (6). As a result two relay control variables, $u_{d}, u_{q}$ defined by (19)-(23), guarantee robustness property in the sliding mode. If the integral part of the error functions is available, the sliding mode starts earlier if compared with the classical one and the equivalent control could be used for the VSI control.

\section{Decomposed SMC System Verification}

Proposed decomposed SMC strategy was verified in two steps. As the first step control of the plant, which consists of PMSM and flexible load, was verified by simulations. Secondly the strategy was applied to the PMSM only for control of its shaft position. In spite of some problem with generation of the observed rotor acceleration, which is necessary as one derivative feedback, this control structure was verified experimentally.
Overall block diagram of decomposed sliding mode control of the drive with IPMSM and flexible coupling is shown in Figure 7. To avoid control chattering, a smoothing integrator is introduced into magnetic flux and motor torque control channels, which are controlled separately. The main goal of linkage magnetic flux control is to comply with vector control condition keeping stator current component $i_{d}=$ 0 . The goal of torque control channel is to reach and keep desired position of the load angle in spite of flexible coupling and presence of external disturbances on the rotor and load side.

The feedback derivatives gains as well as ideal position trajectory, $\theta_{\text {id }}(t)$ computation are based on Dodds settling time formulae [24]:

$$
\frac{\theta_{L}(s)}{\theta_{L z}(s)}=\left[\frac{1}{1+\left(s T_{s s} / 1.5(n+1)\right)}\right]^{n},
$$




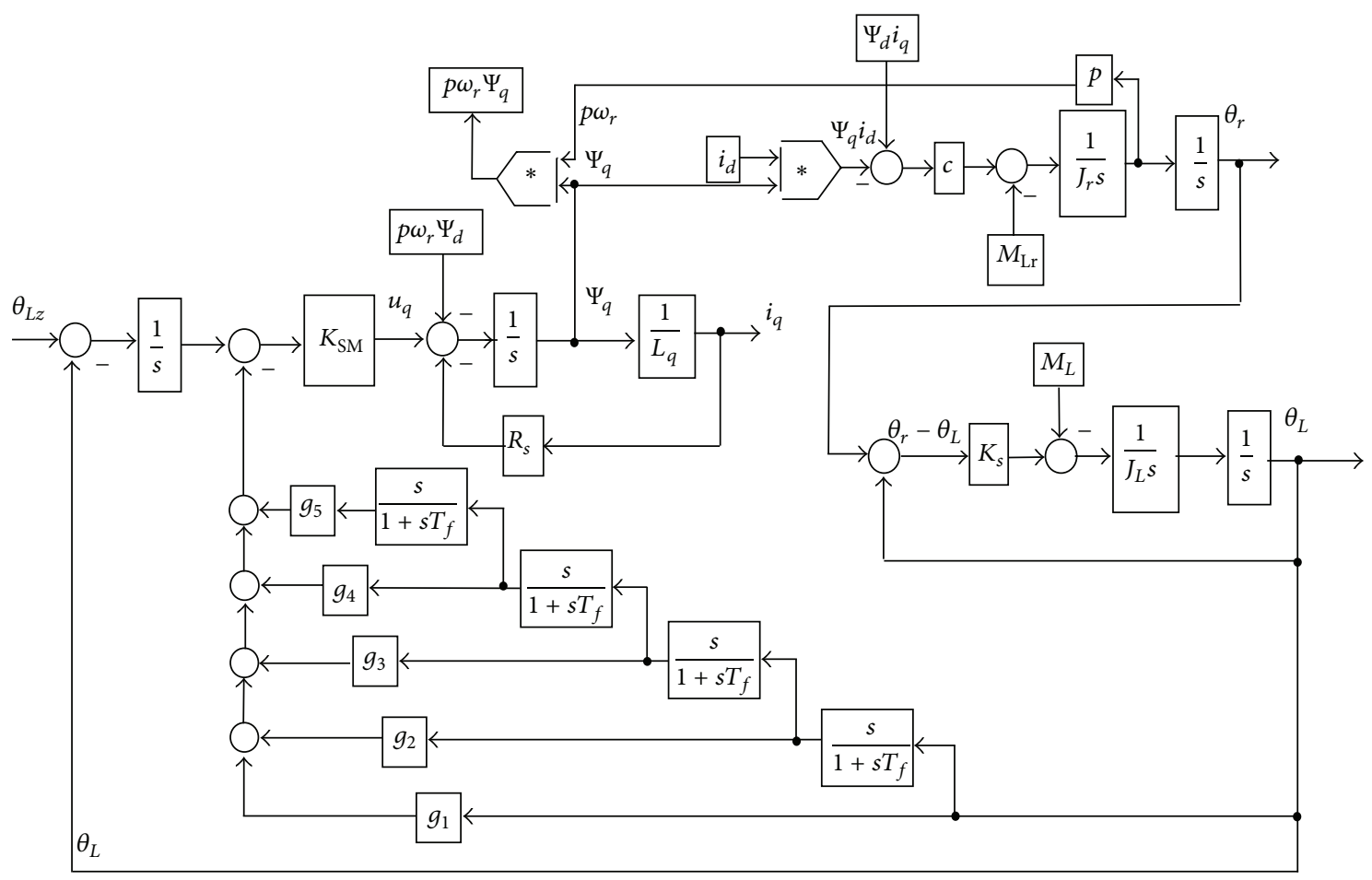

FIGURE 8: Block diagram of modified decomposed SMC of torque channel.

where $n$ is order of the system in sliding mode and $T_{s s}$ is prescribed settling time for given control channel. The magnetic flux control channel including smoothing integrator is 2 nd order; therefore, corresponding to above theory only one derivative feedback is required. Gain, $G_{1}$, of flux channel derivative feedback with settling time, $T_{s \Psi}$, is determined using (30) for $n=1$ as $G_{1}=T_{s \Psi} / 3$. The order of the torque control channel is the sixth; therefore, five derivatives are needed as feedback. Gains, $g_{i}(i=1,2, \ldots, 5)$ of torque channel derivative feedbacks with settling time, $T_{\mathrm{s} \theta}$, are determined using (30) for $n=5$ as $g_{1}=5 T_{s \theta} / 9, g_{2}=10 T_{s \theta}^{2} / 81$, $g_{3}=10 T_{s \theta}^{3} / 729, g_{4}=5 T_{s \theta}^{4} / 6561, g_{5}=T_{s \theta}^{5} / 59049$.

As possible modification of the proposed control system the signum function is replaced by a transfer characteristic consisting of a proportional high gain with saturation, which changes the switching boundary to a boundary layer.

Operation in the modified sliding mode implies operation on the linear, high gain characteristic enabling rearrangement of the block diagrams (valid for both control channels) to avoid the need of the highest output derivative. After this rearrangement the flux control channel operation is based on the integration of error between demanded and real current, $i_{d}(t)$ and one of its feedback with gain, $G_{1}$ from real current, $i_{d}(t)$. Block diagram of modified sliding mode control for torque control channel shows Figure 8.

The parameters of the IPMSM and load are listed in the Appendix. For whole simulation interval shaft of the motor is loaded with constant friction torque, $M_{L r}=0,5 \mathrm{Nm}$. The external load torque, $M_{L}=4 \mathrm{Nm}$ is applied at $t=0.5 \mathrm{~s}$ and for load angle control simulated as a step change, being zero for the time interval $t<0.5 \mathrm{~s}$. Computational step of simulations was $h_{s}=1 \mu \mathrm{s}$ for correct simulation of PWM, while the sampling frequency for control algorithm was $1 / h_{c}=0.1 \mathrm{~ms}$, which corresponds to the sampling frequency achieved during previous experiments sliding mode control of the IPMSM rotor position.

All the simulations are carried out with zero initial state variables and a step load position demand, $\theta_{L z}=2 \pi \mathrm{rad}$. Prescribed settling time of magnetic flux channel was $T_{s \theta}=$ $20 \mathrm{~ms}$ and settling time of motor torque channel was chosen as $T_{s \theta}=0.25 \mathrm{~s}$. Error function between prescribed dynamic computed using (30) and achieved dynamic is evaluated as $e_{\theta}(t)=\theta_{\text {id }}(t)-\theta_{r}(t)$, (in plots 2-times magnified). Simulation results for both designed control systems in sliding mode are arranged in the same pattern of subplots in Figures 9 and 10.

Subplots (a) show demanded voltages $u_{d z}(t)$ and $u_{q z}(t)$ in the rotor fixed frame $(d, q)$, which produce in corresponding windings currents capable to keep constant magnetic flux and to developed motor torque achieving prescribed dynamics and capable to counteract friction torque of motor and external load torque. Subplots (b) show demanded voltages $u_{\alpha z}(t)$ and $u_{\beta z}(t)$ in the stator fixed frame $(\alpha, \beta)$. These are effective outputs of the control algorithm and after transformation into three-phase system and PWM sampled serve to supply IPMSM terminals.

Demanded current $i_{d z}$ and $i_{q z}$ in the rotor fixed frame $(d, q)$ generated by control algorithms are shown in subplots (c). As can be seen $i_{d z}(t)$ is kept close to zero, as required for the vector control of IPMSM. Initial positive and negative swings of $i_{q z}(t)$ current produce the acceleration and 


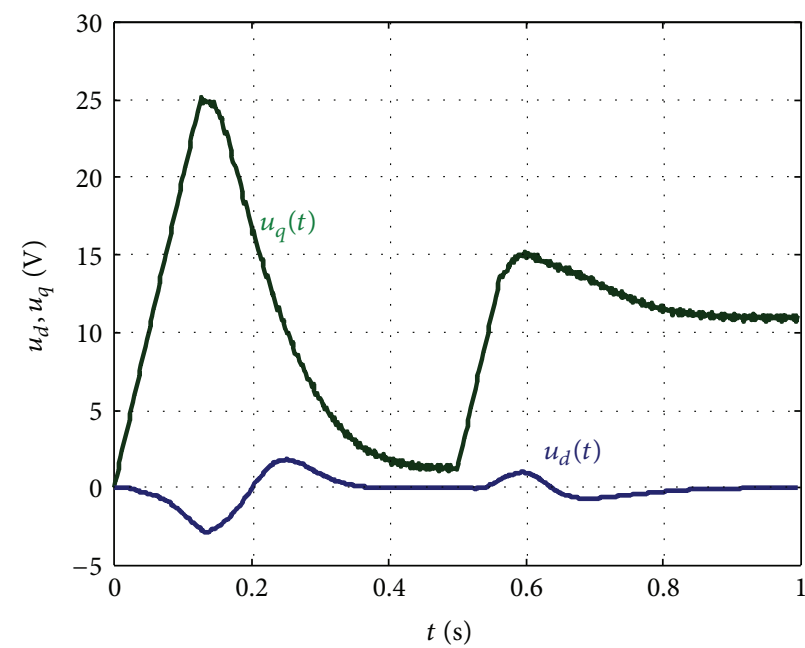

(a) Demanded voltages $(d, q)$

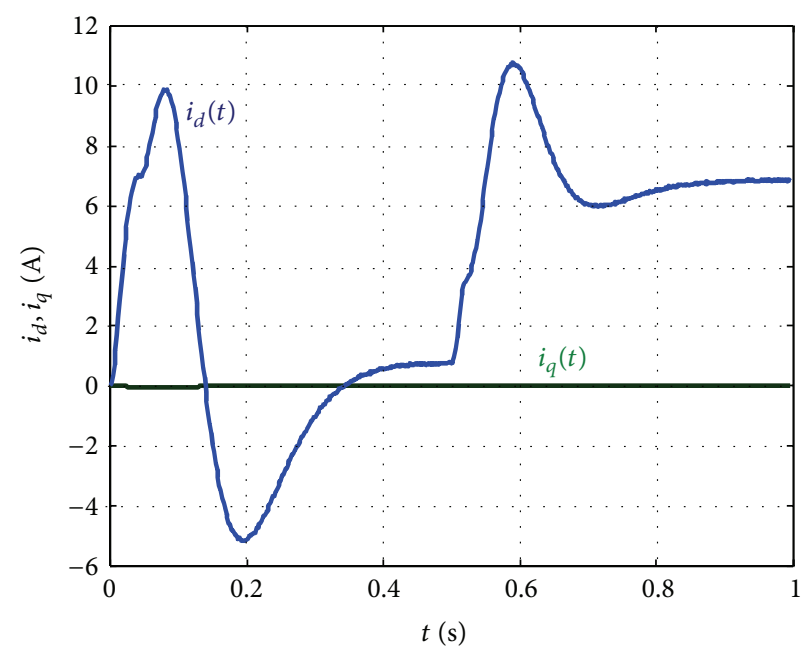

(c) Demanded currents $(d, q)$

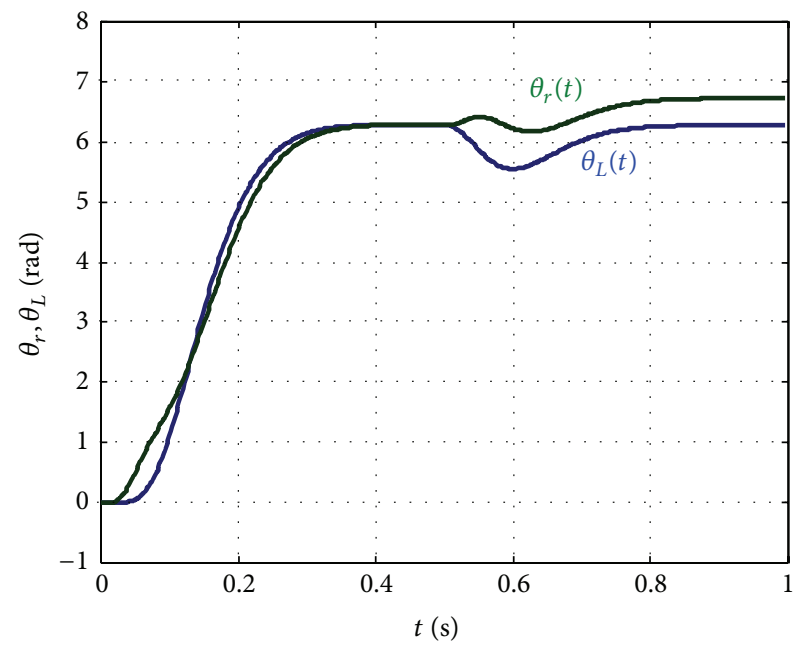

(e) Load and rotor position

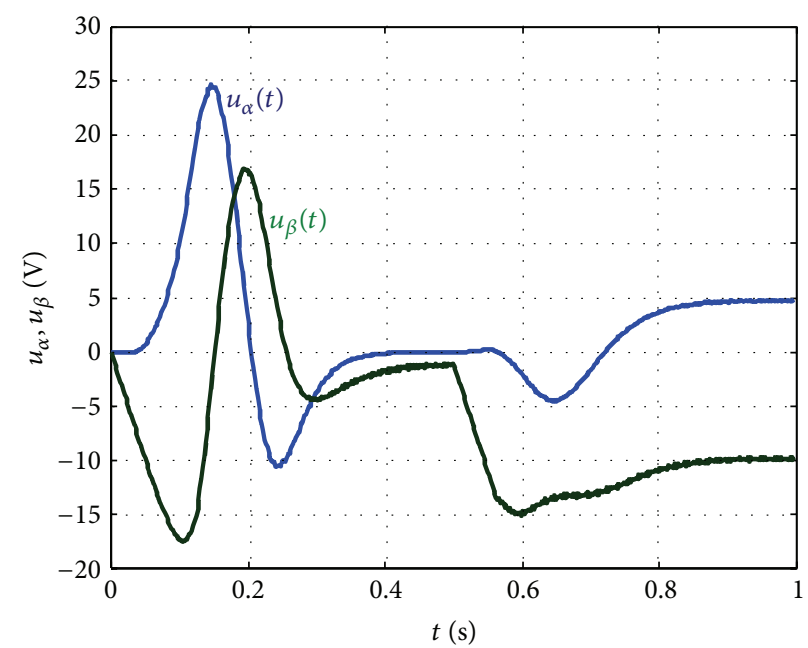

(b) Demanded voltages $(\alpha, \beta)$

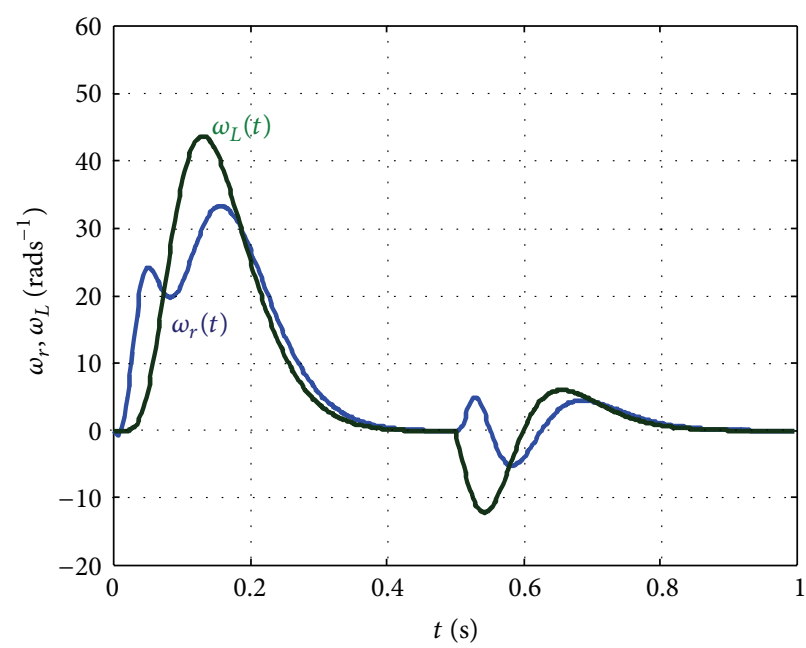

(d) Load and rotor velocity

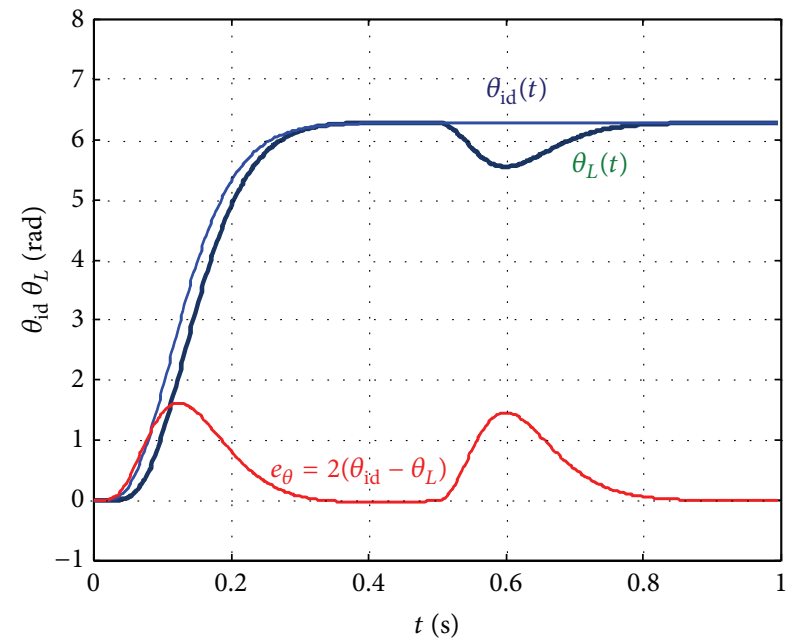

(f) Ideal, load and error position

FIGURE 9: Decomposed SMC system of the drive with PMSM and flexible coupling. 


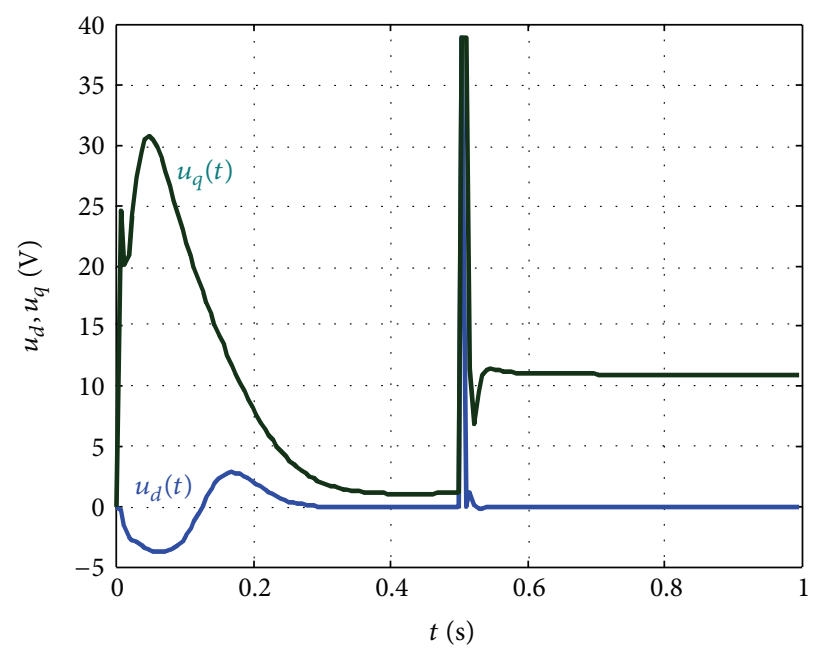

(a) Demanded voltages $(d, q)$

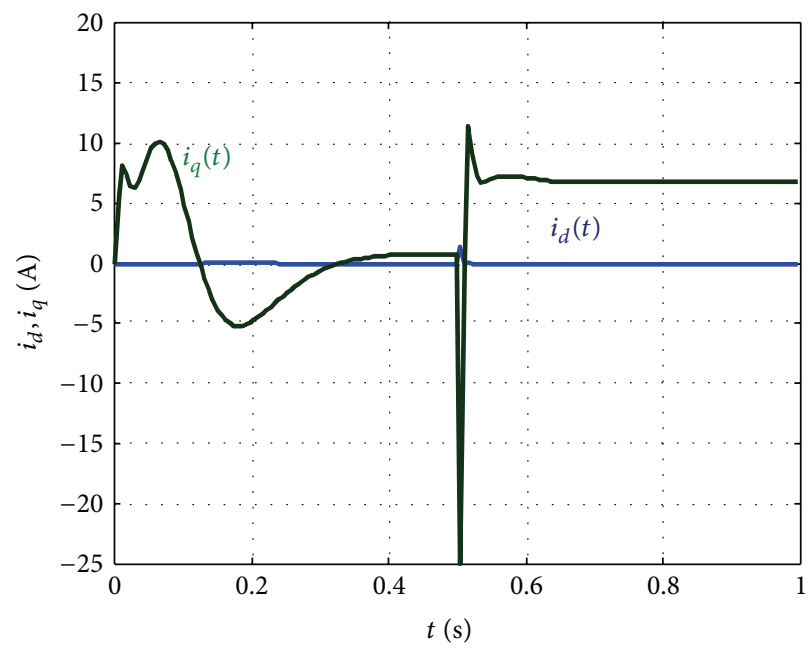

(c) Demanded currents $(d, q)$

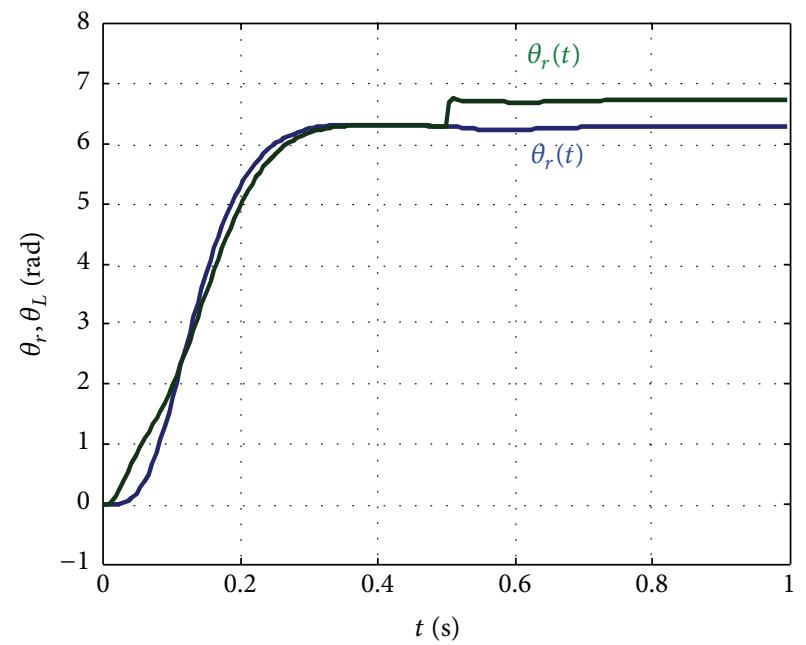

(e) Load and rotor position

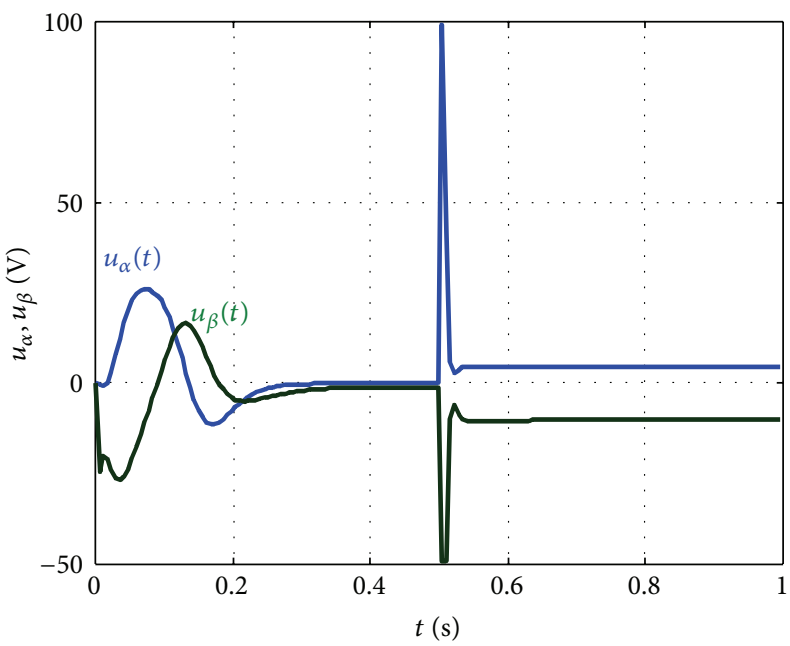

(b) Demanded voltages $(\alpha, \beta)$

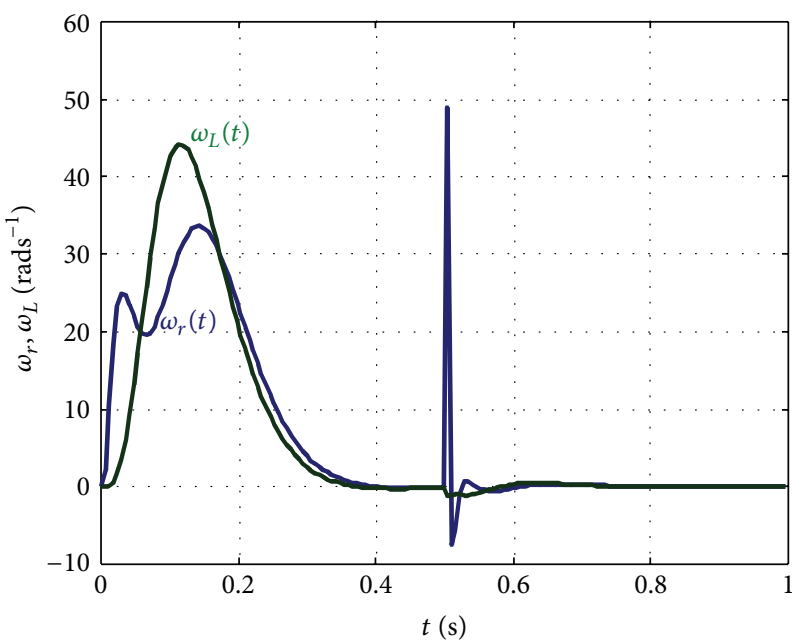

(d) Load and rotor velocity

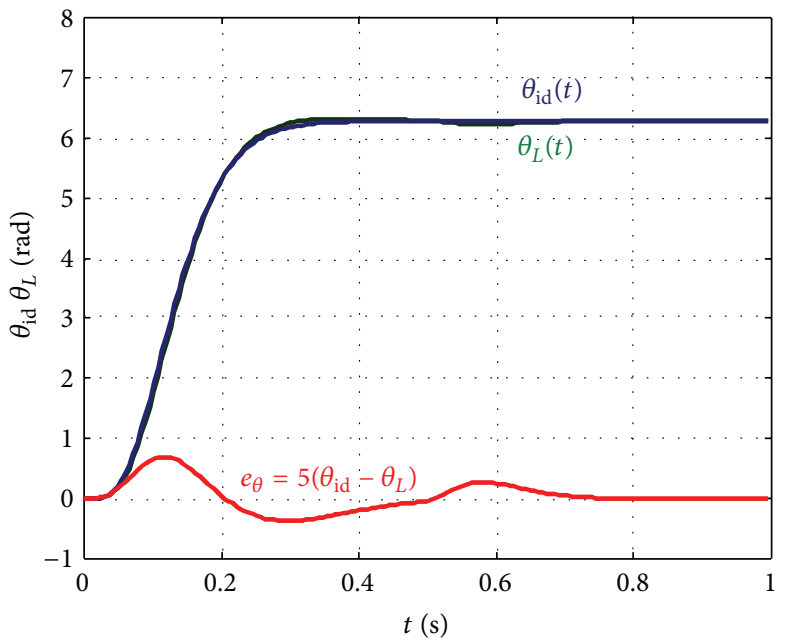

(f) Ideal, load and error position

FIGURE 10: Modified control system of the drive with PMSM and flexible coupling. 


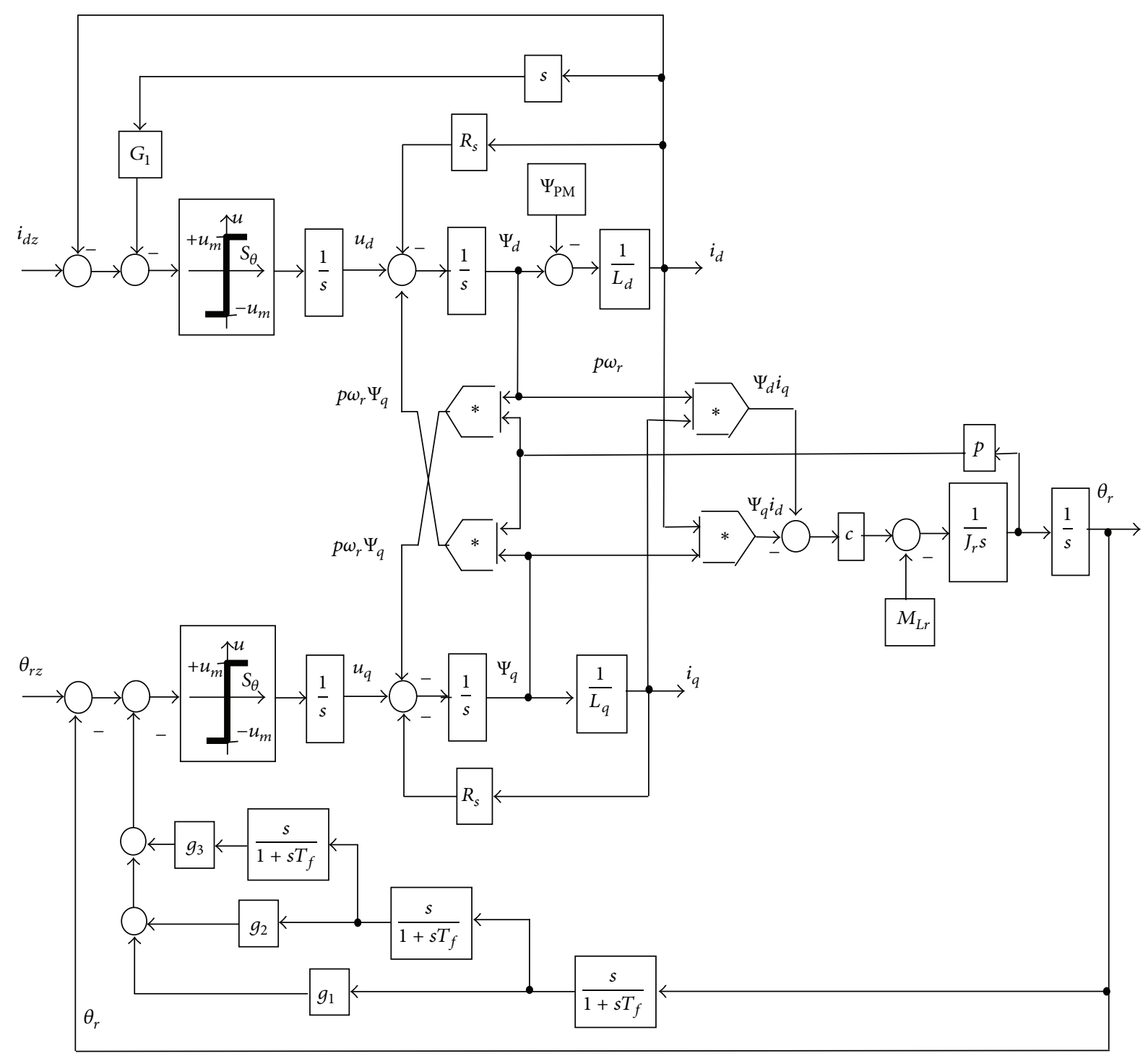

FIGURE 11: Overall block diagram of decomposed SMC of IPMSM rotor position.

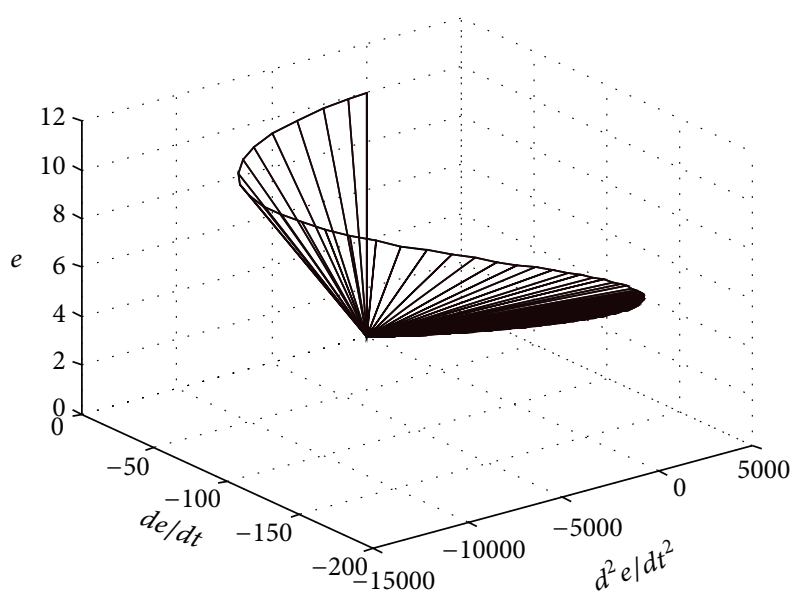

(a)

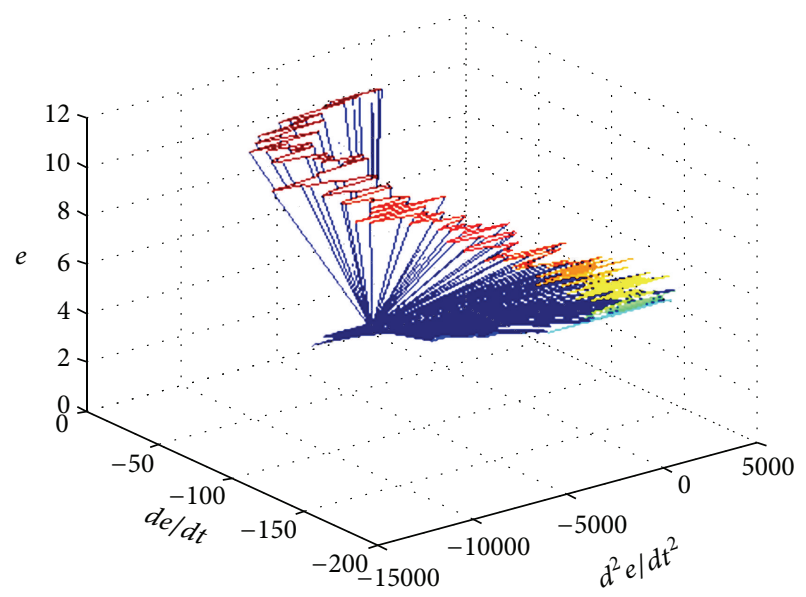

(b)

FIGURE 12: Designed and measured switching surface for decomposed SMC of PMSM rotor angle. 


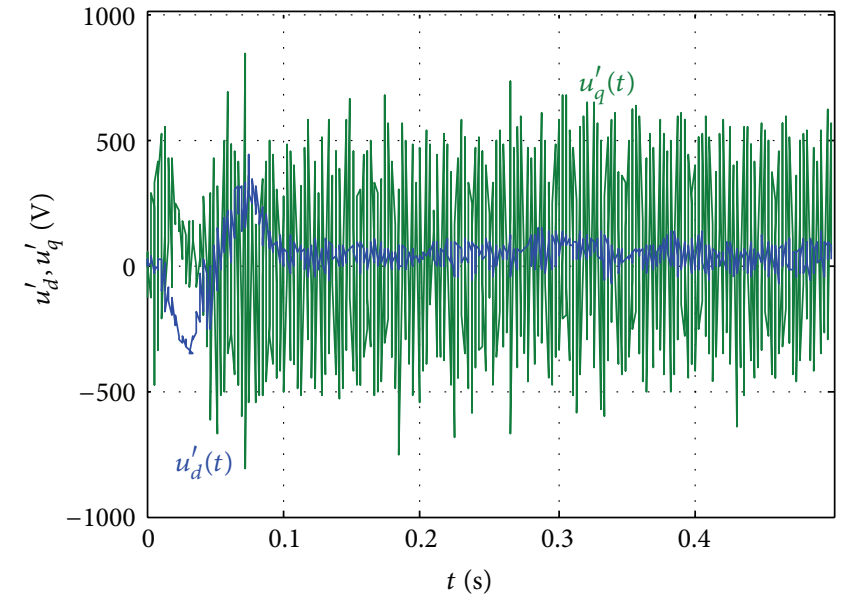

(a) Applied stator $d_{-} q$ voltages

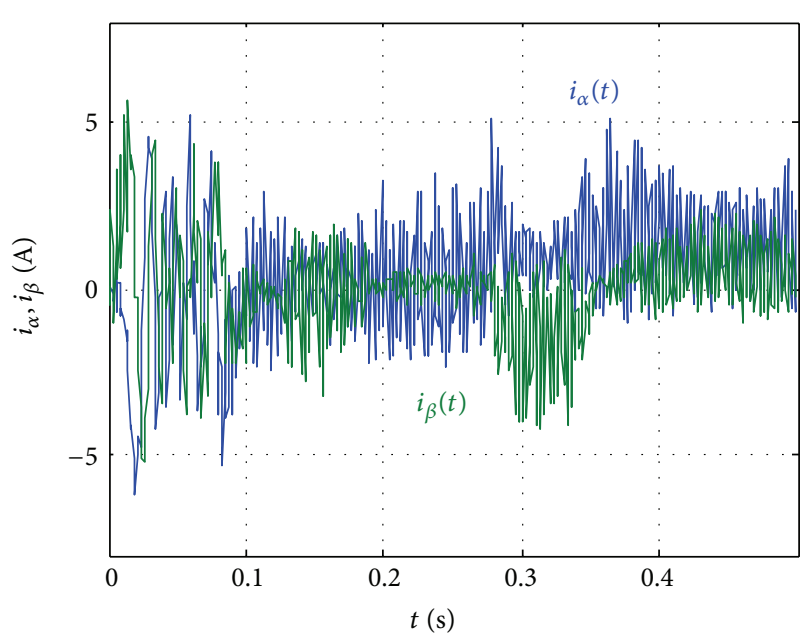

(c) Stator $\alpha_{-} \beta$ current components

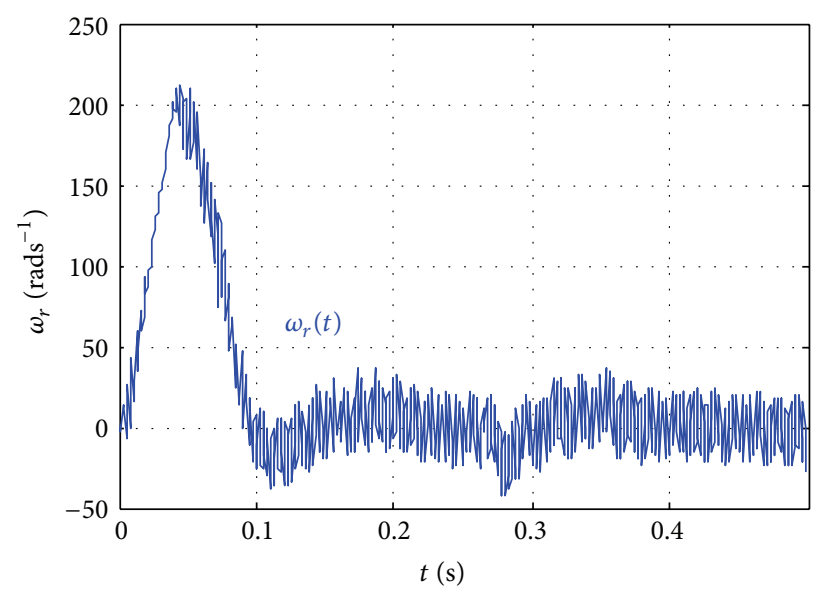

(e) Rotor angular velocity

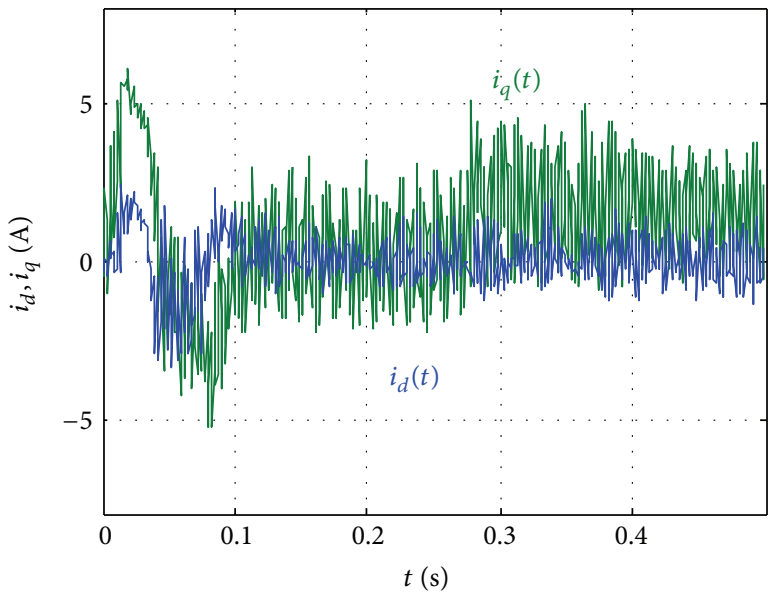

(b) Stator $d_{-} q$ current components

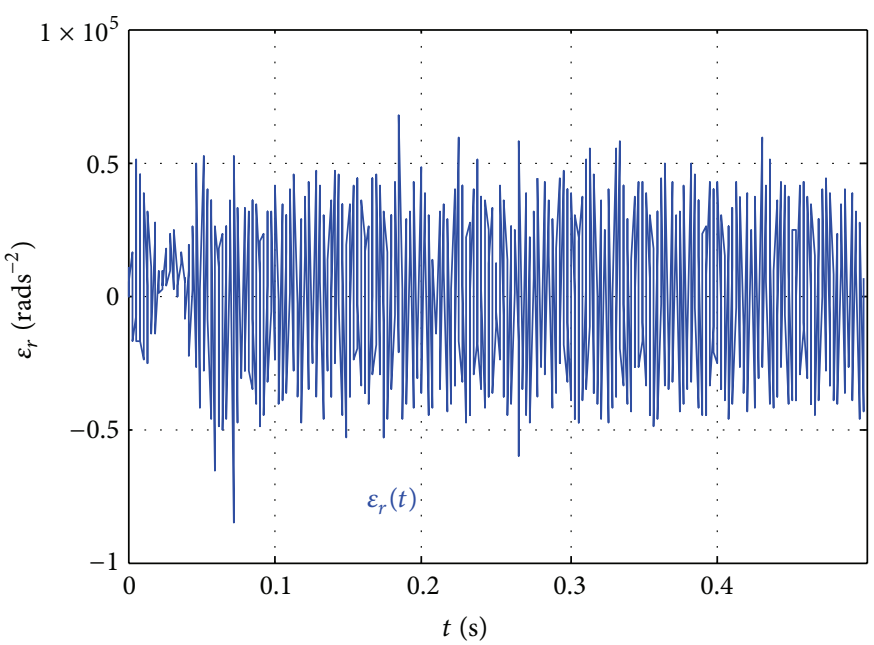

(d) Rotor angular acceleration

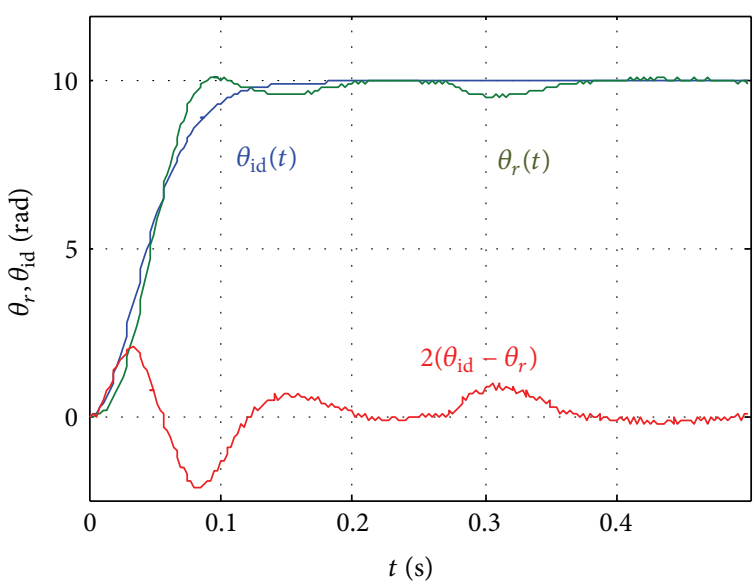

(f) Rotor and ideal position response

FIGURE 13: Preliminary experimental results for decomposed SMC of PMSM rotor angle. 
deceleration torques, which can be followed together with the increase of $i_{q z}(t)$ after $t=0.5$ s counteracting the external load torque transmitted via the spring.

The experimental results for decomposed SMC of rotor angle are shown in Figure 13. As expected at both control algorithms the acceleration period is followed by the deceleration one, to achieve prescribed response. Subplots (e) show the positions of the rotor and the load. The compensation of the external load torque applied at $t=0.5 \mathrm{~s}$ is clearly evident from the angular displacement between the IPMSM rotor and the load after this torque is applied. This entails a constant torsional deflection of the spring, which is clear from the constant difference between $\theta_{R}$ and $\theta_{\mathrm{L}}$ occurring just after the load torque achieves its steady state. The initial differences between the rotor and load displacements are due to the acceleration and deceleration torques being applied via the torsion spring.

Finally, subplots (f) of both figures show the ideal response and response of the control system to the step load position demand, $\theta_{L z}=2 \pi \mathrm{rad}$. including magnified difference between them $(2 x$ and $5 x)$. The realisation of the prescribed position dynamics is nearly accurate, which is clear from the magnified differences. Also the prescribed settling time for position control is kept well because the load position $\theta_{\mathrm{L}}(t)$ passes through $1.9 \pi$ radian $(95 \%$ of demanded value) at a time close to $0.25 \mathrm{~s}$.

Presented simulation results confirm possibility of decomposed sliding mode control of the load angle in spite of flexible coupling between motor and load. As can be seen from Figures 9 and 10, subplots (f), the load angle responses follow the prescribed ideal response fairly closely. Comparison of both simulations shows a good agreement with the theoretical predictions made during both decomposed SMC algorithms development.

To avoid problem with high order derivatives the decomposed SMC strategy was applied to PMSM for rotor position control [25]. Overall control system block diagram is shown in Figure 11. Design of flux control channel is the same as it was described for decomposed SMC of PMSM with flexible coupling. The torque control channel is in this case the fourth order; therefore, three derivatives of controlled variable are needed as feedback. Gains, $g_{i}(i=1,2,3)$ of torque channel derivative feedbacks with settling time, $T_{\mathrm{s} \theta}$, are determined using (30) as $g_{1}=T_{s \theta} / 2, g_{2}=T_{s \theta}^{2} / 12, g_{3}=T_{s \theta}^{3} / 216$. Designed and measured switching surfaces for PMSM rotor position control are shown in Figure 12.

The experimental results for decomposed SMC of rotor angle (for the idle running PMSM) are shown in Figure 13. Nearly constant load torque was developed by locked torque of the induction machine and applied at $t=0.27 \mathrm{~s}$. The individual subplots (a)-(c) show the applied stator voltage components and corresponding stator current components, respectively. Subplot (d) and subplot (e) show the rotor angular acceleration and rotor speed and as the outputs of the observer taken as the inputs of SMC law. Subplot (f) shows response of the control system together with the designed ideal response to a step position demand of $\theta_{r \mathrm{dem}}=10 \mathrm{rad}$ including the 2 times magnified difference between them.
Comparison of PMSM with flexible coupling simulations and preliminary experiments with decomposed SMC of PMSM rotor position show a good agreement with the theoretical predictions made during the control system development. If suitable observer of control variables is developed it is highly desirable to investigate proposed decomposed SMC strategy experimentally.

\section{Conclusions}

The simulations and preliminary experiment presented predict that the proposed decomposed sliding mode control system and its modification can be made to follow the ideal closed loop dynamics of the load angle with moderate accuracy in spite of flexible coupling between motor and load. As a result, this is acceptable considering that no motor or driven load parameters were needed for control system design.

Some variables of modified control system show significant overshoots during transients, which were limited in subplots due to graphic purposes. In spite of very close tracking of ideal position modified control system shows also small overshoots of load position, which is not acceptable for position control systems requiring high precision.

The results obtained are sufficiently promising to warrant further experimental trials, which were not carried out due to problems with output derivatives of higher order. For the future work derivatives estimation methods such as observer with filtering properties or high gain multiple integrator observer together with measurement of load position with higher accuracy are recommended.

\section{Appendix}

Parameters of PMSM are winding resistance, $R_{s}=1.3 \Omega$, winding inductances, $L_{d}=14.4 \mathrm{mH}, L_{q}=16.3 \mathrm{mH}$, permanent magnet flux, $\Psi_{\mathrm{PM}}=0.13 \mathrm{~Wb}$, number of pole pair, $p=5$, and moment of inertia, $J_{r}=0.00063 \mathrm{~kg} \cdot \mathrm{m}^{2}$.

Load parameters are load moment of inertia, $J_{L}=$ $0.000918 \mathrm{kgm}^{2}$, and shaft spring constant, $K_{s}=9 \mathrm{Nrad}^{-1}$.

\section{Acknowledgment}

The authors wish to thank Slovak Grant Agency VEGA for funding Project no. 1/0355/11 "Optimal Control Techniques for Decreasing Losses of A.C. Drives."

\section{References}

[1] Y. Hori, H. Sawada, and Y. Chun, "Slow resonance ratio control for vibration suppression and disturbance rejection in torsional system," IEEE Transactions on Industrial Electronics, vol. 46, no. 1, pp. 162-168, 1999.

[2] S. Katsura and K. Ohnishi, "Force servoing by flexible manipulator based on resonance ratio control," in Proceedings of the IEEE International Symposium on Industrial Electronics (ISIE '05), pp. 1343-1348, Dubrovnik, Croatia, June 2005. 
[3] S. Ryvkin, D. Izosimov, and S. Baida, "Flex mechanics digital control design," in Proceedings of the IEEE International Conference on Industrial Technology (ICIT '03), vol. 1, pp. 298-303, Maribor, Slovenia, December 2003.

[4] K. Szabat and T. Orlowska-Kowalska, "Vibration suppression in a two-mass drive system using PI speed controller and additional feedbacks-comparative study," IEEE Transactions on Industrial Electronics, vol. 54, no. 2, pp. 1193-1206, 2007.

[5] M. A. Valenzuela, J. M. Bentley, A. Villablanca, and R. D. Lorenz, "Dynamic compensation of torsional oscillation in paper machine sections," IEEE Transactions on Industry Applications, vol. 41, no. 6, pp. 1458-1466, 2005.

[6] M. Vasak, M. Baotic, I. Petrovic, and N. Peric, "Hybrid theorybased time-optimal control of an electronic throttle," IEEE Transactions on Industrial Electronic, vol. 43, no. 3, pp. 14831494, 2007.

[7] T. M. O'Sullivan, C. M. Bingham, and N. Schofield, "Enhanced servo-control performance of dual-mass systems," IEEE Transactions on Industrial Electronics, vol. 54, no. 3, pp. 1387-1399, 2007.

[8] G. Zhang and J. Furusho, "Speed control of two-inertia system by PI/PID control," IEEE Transactions on Industrial Electronics, vol. 47, no. 3, pp. 603-609, 2000.

[9] A. N. Moser, "Designing controllers for flexible structures with H-infinity/ $\mu$-synthesis," IEEE Transactions on Control Systems, vol. 13, no. 2, pp. 79-89, 1993.

[10] M. Cychowski, K. Szabat, and T. Orlowska-Kowalska, "Constrained model predictive control of the drive system with mechanical elasticity," IEEE Transactions on Industrial Electronics, vol. 56, no. 6, pp. 1963-1973, 2009.

[11] J. M. Maciejowski, Predictive Control with Constraints, Prentice Hall, London, UK, 2002.

[12] J. Wang, Y. Zhang, L. Xu, Y. Jing, and S. Zhang, "Torsional vibration suppression of rolling mill with constrained model predictive control," in Proceedings of the 6th World Congress on Intelligent Control and Automation (WCICA '06), pp. 64016405, Dalian, China, June 2006.

[13] T. Orlowska-Kowalska and K. Szabat, "Damping of torsional vibrations in two-mass system using adaptive sliding neurofuzzy approach," IEEE Transactions on Industrial Informatics, vol. 4, no. 1, pp. 47-57, 2008.

[14] S. J. Dodds and J. Vittek, "Sliding mode control of PMSM drives subject to torsion oscillations in the mechanical load," in Proceedings of the 13th International Power Electronics and Motion Control Conference (EPE-PEMC '08), pp. 2551-2558, Poznan, Poland, September 2008.

[15] V. Utkin, J. Guldner, and J. Shi, Sliding Mode Control in ElectroMechanical Systems, CRC Press, 2nd edition, 2009.

[16] S. Ryvkin and E. P. Lever, Sliding Mode Control for Synchronous Electric Drives, CRC press, 2011.

[17] N. Mohan, T. M. Undeland, and W. P. Robbins, Power Electronics: Converters, Applications and Design, John Wiley \& Sons, New York, NY, USA, 3rd edition, 2003.

[18] V. A. Kotelnikov, "On the carrying capacity of the ether and wire in telecommunications," in Proceedings of the 1st All-Union Conference on Questions of Communication, Moscow, Russia, January 1933, (Russian).

[19] R. J. Marks, II, Introduction to Shannon Sampling and Interpolation Theory, Springer Texts in Electrical Engineering, Springer, New York, NY, USA, 1991.
[20] S. E. Ryvkin and D. B. Izosimov, "Comparison of pulsewidth modulation algorithms for three-phase voltage inverters," Electrical Technology, no. 2, pp. 133-144, 1997.

[21] G. S. Zinoviev, Power Electronics Backgrounds, Publishing House of Novosibirsk State Technical University, Novosibirsk, Russia, 2005, (Russian).

[22] W. Leonhard, Control of Electrical Drives, Springer, Berlin, Germany, 2001.

[23] P. Brandstetter and T. Krecek, "Speed and current control of permanent magnet synchronous motor drive using IMC controllers," Advances in Electrical and Computer Engineering, no. 4, pp. 3-10, 2012.

[24] S. J. Dodds, "Settling time formulae for the design of control systems with linear closed loop dynamics," in Proceedings of the 3rd Annual Conference on Advances in Computing and Technology (AC\&T '08), pp. 31-39, The School of Computing and Technology, University of East London, London, UK, 2008.

[25] J. Vittek, S. J. Dodds, P. Briš, M. Štulrajter, and P. Makyš, "Experimental verification of chattering free sliding mode control of the drive position employing PMSM," Journal of Electrical Engineering, vol. 59, no. 3, pp. 139-145, 2008. 


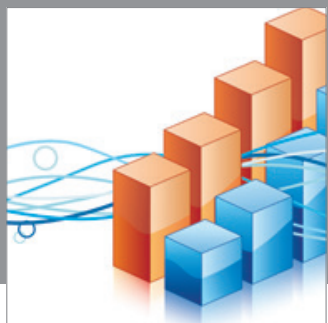

Advances in

Operations Research

mansans

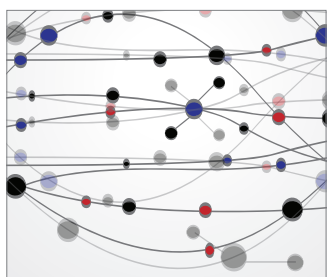

The Scientific World Journal
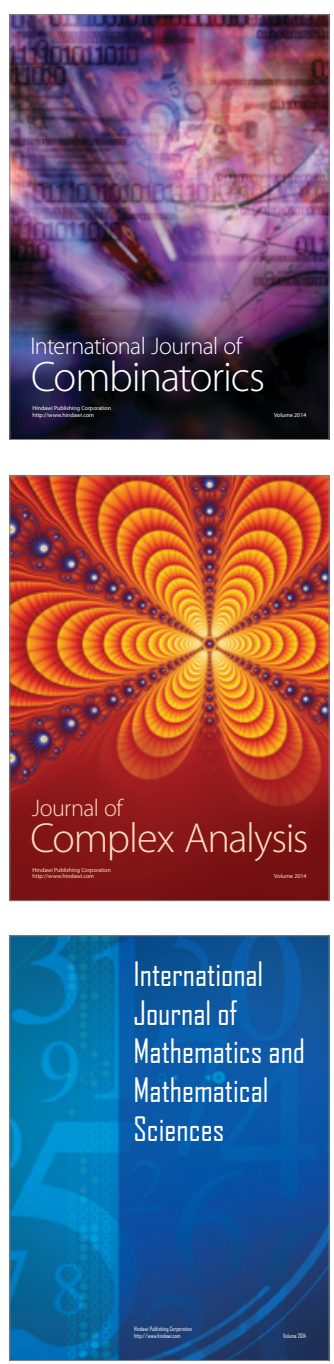
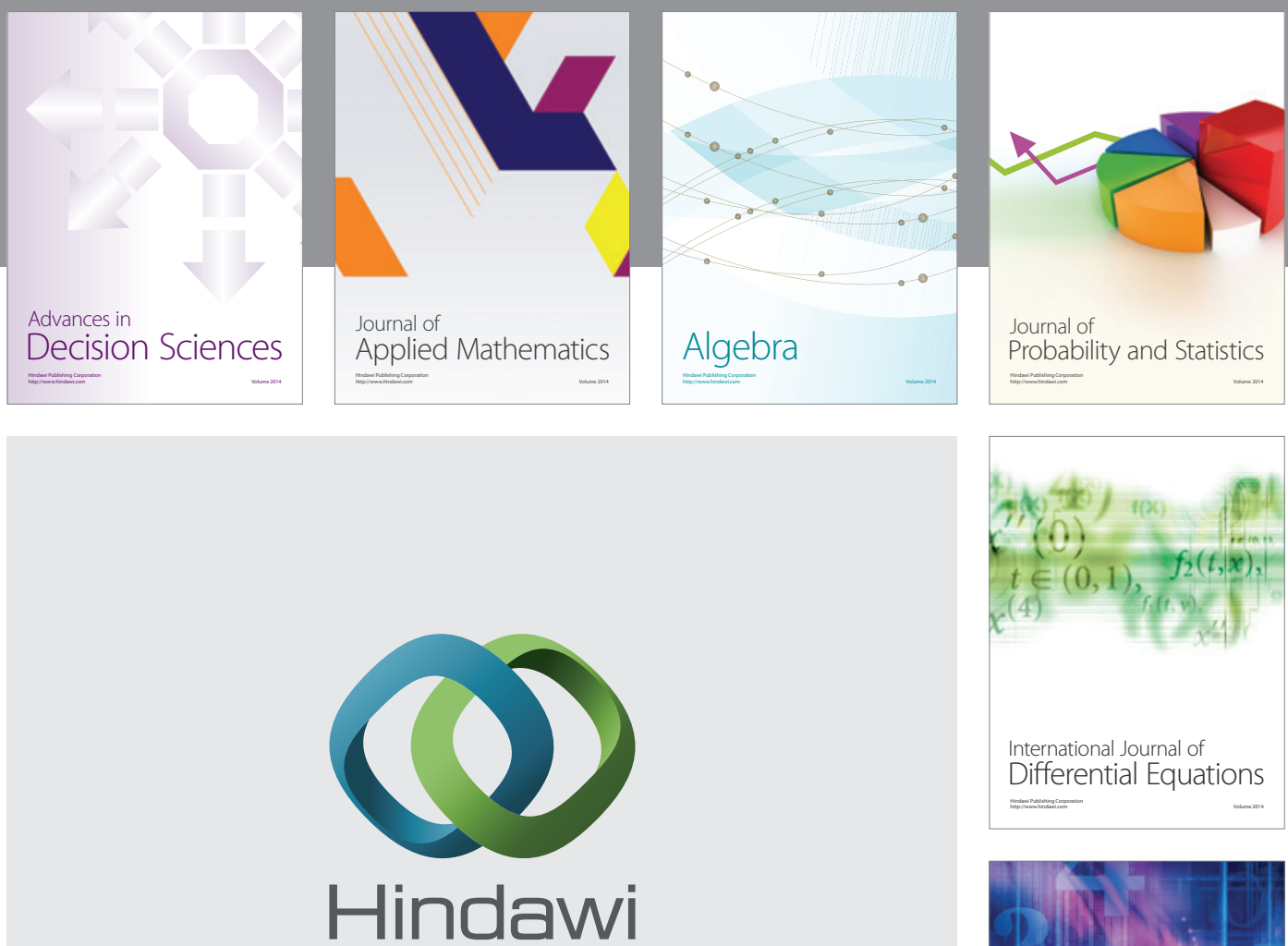

Submit your manuscripts at http://www.hindawi.com
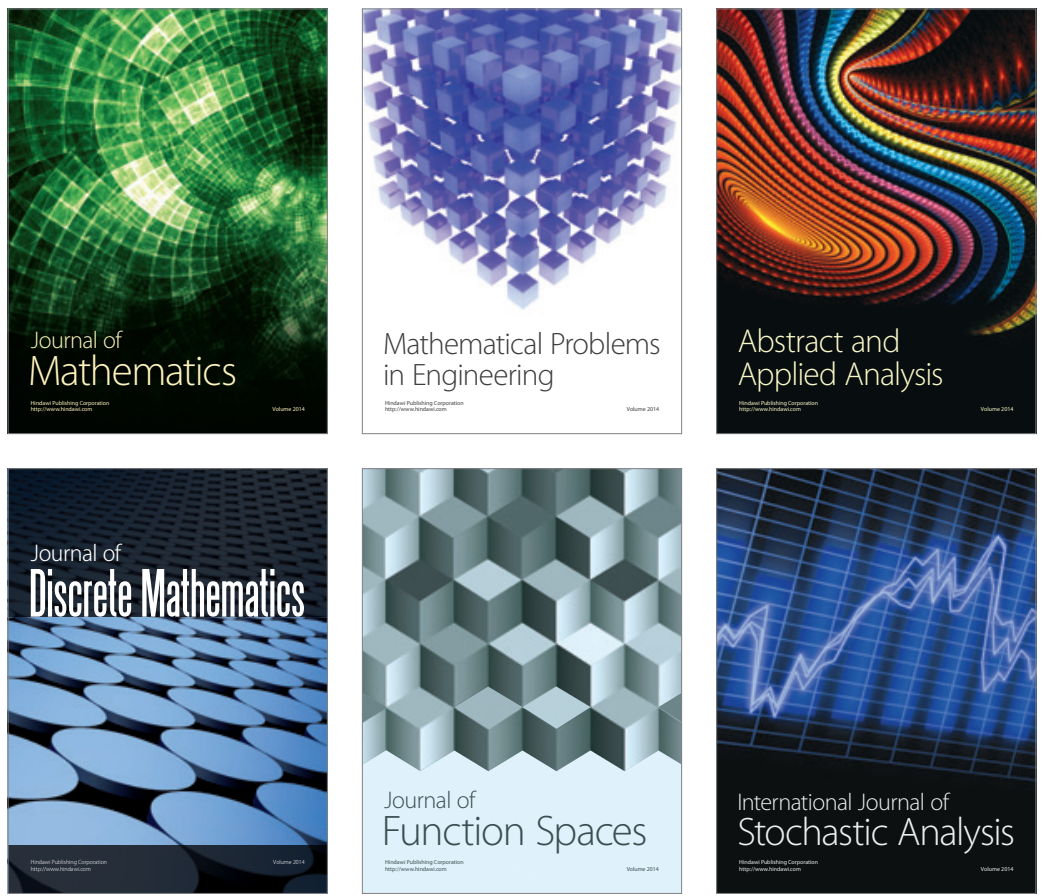

Journal of

Function Spaces

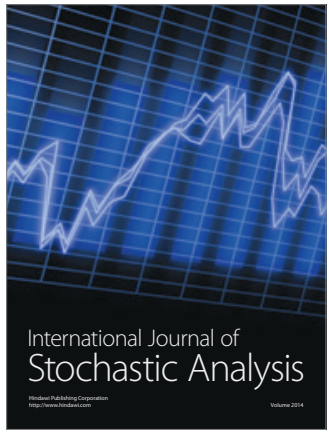

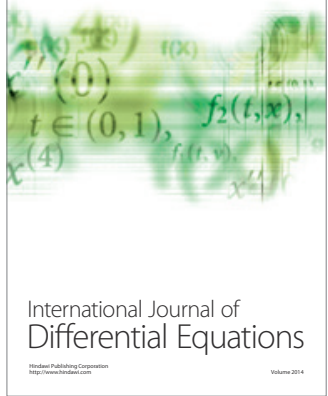
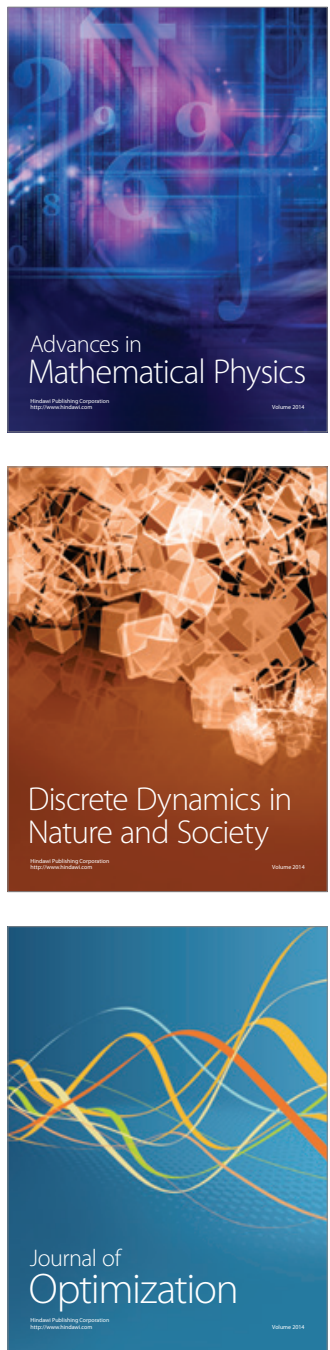